LVOV MATHEMATICAL SCHOOL

IN THE PERIOD 1915-45 AS SEEN TODAY

BANACH CENTER PUBLICATIONS, VOLUME 87

INSTITUTE OF MATHEMATICS

POLISH ACADEMY OF SCIENCES

WARSZAWA 2009

\title{
DIFFERENTIAL AND INTEGRAL CALCULUS FOR A SCHAUDER BASIS ON A FRACTAL SET (I) (SCHAUDER BASIS 80 YEARS AFTER)
}

\author{
JULIAN ŁAWRYNOWICZ \\ Institute of Physics, University of Eódź \\ Pomorska 149/53, PL-90-236 Eódź \\ Institute of Mathematics, Polish Academy of Sciences \\ Łódź Branch, Banacha 22, PL-90-238 Eódź, Poland \\ E-mail: jlawryno@uni.lodz.pl \\ TATSURO OGATA and OSAMU SUZUKI \\ Department of Computer and System Analysis \\ College of Humanities and Sciences, Nihon University \\ Sakurajosui 3-25-40, 156-8550 Setagaya-ku,Tokyo, Japan \\ E-mail:togata@cssa.chs.nihon-u.ac.jp; osuzuki@cssa.chs.nihon-u.ac.jp
}

\begin{abstract}
In this paper we introduce a concept of Schauder basis on a self-similar fractal set and develop differential and integral calculus for them. We give the following results:

(1) We introduce a Schauder/Haar basis on a self-similar fractal set (Theorems I and I').

(2) We obtain a wavelet expansion for the $L^{2}$-space with respect to the Hausdorff measure on a self-similar fractal set (Theorems II and II').

(3) We introduce a product structure and derivation on a self-similar fractal set (Theorem III).

(4) We give the Taylor expansion theorem on a fractal set (Theorem IV and IV').

(5) By use of the Taylor expansion for wavelet functions, we introduce basic functions, for example, exponential and trigonometrical functions, and discuss the relationship between the usual and introduced corresponding special functions (Theorem V).

(6) Finally we discuss the relationship between the wavelet functions and the generating functions of the dynamical systems on a fractal set and show that our wavelet expansions can describe several fluctuations observed in nature.
\end{abstract}

2000 Mathematics Subject Classification: Primary 37F45; Secondary 28A80.

Research of the first author was partially supported by the Ministry of Science and Higher Education grant PB1 P03A 00126 (Sections 1-5 of the paper), and partially by the grant of the University of Łódź no. 505/692 (Sections 6-11).

The paper is in final form and no version of it will be published elsewhere. 
1. Introduction. In 1927, Schauder [10, 11] introduced a system of functions which is called the Schauder basis on the interval and made a uniform approximation of a continuous function. In this paper we shall introduce a concept of Schauder basis on a self-similar fractal set and make differential and integral calculus for them. Hence we may say that Schauder's observation will supply an important method for new fields of mathematics still now and furthermore in the future.

Fractal structure can be observed in many places and it is expected that it will supply an important method for complex systems, especially the description of fluctuations in music, stock price behaviour, and neural systems of brains. The fundamental material is already well prepared in the famous book by Falconer [2]: the geometric measure theory with respect to the Hausdorff measure is well exposed and its applications are given. The concept of self-similar fractal sets was introduced by Hutchinson in [3] and the geometric theory of the Hausdorff measure is treated there in detail. Many authors have contributed to the calculus of the Hausdorff dimensions.

On the other hand, the analysis on a fractal set also has started and became one of the most interesting topics. One of important and beatiful results is supplied by the theory of Laplacian on the Sierpiński's gasket [3]. We may expect further developments in this direction.

In this paper we introduce differential and integral calculus on a self-similar fractal set. The basic idea is to introduce a wavelet expansion on a fractal set and to define a derivation on the Hilbert space of $L^{2}$-space with respect to the Hausdorff measures on it. This can be performed by introducing the "*-product" on the space. Then we can see that the derivation can be defined by the shift operator on the space and develop differential calculus on a self-similar fractal set with the Taylor expansion theorem on it. By use of the Taylor expansion, we can introduce basic functions, for example, exponential, trigonometrical, and geometric functions on a fractal set. Here we wish to stress that we can give an embedding theorem of the usual corresponding functions into our basic functions on the fractal sets which may be regarded as the noncommutative embedding of derivations. We call such an embedding the fluctuation mapping. By this mapping we can get functions with complicated fluctuations from smooth functions without fluctuations. We can find a new approach to the problem of the basic fluctuations in the nature by such a mapping $[6,8]$.

This is the first part of our study on a fractal set. In the second part we shall introduce differential forms and chains on fractal sets and develop the vector anlysis on a fractal set.

2. Self-similar fractal set. In this section we recall some basic material on fractal sets $[2,3]$. Here we restrict ourselves only to a system of self-similar mappings $\left\{\sigma_{j}: j=\right.$ $1,2, \ldots, N\}$ of a compact set $K_{0}$. Then we can see that there exist positive numbers $\lambda_{i}$ $\left(0<\lambda_{i}<1\right)$ satisfying the conditions:

$$
d\left(\sigma_{i}(x), \sigma_{i}(y)\right)=\lambda_{i} d(x, y) \quad(i=1,2, \ldots, N) .
$$

Then we give the definition of a self-similar fractal set: 
Definition 1 (self-similar fractal set). For a system of self-similar mappings $\sigma_{j}: K_{0} \rightarrow$ $K_{0}(j=1,2, \ldots, N)$, we put

$$
K=\bigcap_{n=1}^{\infty} K_{n}, \text { where } K_{n}=\bigcup_{j=1}^{N} \sigma_{j}\left(K_{n-1}\right),
$$

and call $K$ a self-similar fractal set.

In this paper we always assume the following separation condition:

Definition 2 (separation condition). A system of mappings $\sigma_{j}: K_{0} \rightarrow K_{0}(j=$ $1,2, \ldots, N)$ is said to satisfy the separation condition whenever

$$
\sigma_{i}\left(\stackrel{\circ}{K_{0}}\right) \cap \sigma_{j}\left(\stackrel{\circ}{K_{0}}\right)=\emptyset \quad(i \neq j)
$$

where $\stackrel{\circ}{K_{0}}$ is the open kernel of $K_{0}$.

Some basic properties of fractal sets. Under the condition (2.1), we observe the following facts:

(1) We have the invariance condition:

$$
K=\bigcup_{j=1}^{N} \sigma_{j}(K)
$$

(2) In the case of a self-similar fractal set, we can calculate the Hausdorff dimension $D\left(=\operatorname{dim}_{H} K\right)$ by the following formula:

$$
\sum_{j=1}^{N} \lambda_{j}^{D}=1
$$

The proof will be given later.

Here we give examples of a fractal set.

EXAMPLE 1 (Cantor set). For a system of self-similar mappings $\sigma_{1}=\frac{1}{3} x, \sigma_{2}=\frac{1}{3} x+\frac{2}{3}$, we choose

$$
C=\bigcap_{n=1}^{\infty} C_{n}
$$

where (see Fig. 1):

$$
\begin{aligned}
& C_{0}=I, \\
& C_{n}=\sigma_{1}\left(C_{n-1}\right) \cup \sigma_{2}\left(C_{n-1}\right) .
\end{aligned}
$$

The Hausdorff dimension $D$ is

$$
D=\log 2 / \log 3
$$

EXAMPLE 2 (self-similar fractal set of Cantor type). In a similar manner we can define a fractal set of Cantor type, removing subintervals from the original interval: Let $I$ be 
the interval $I=[0,1]$ and let $\sigma_{i}: I \rightarrow I(i=1, \ldots, N)$ be a system of contractions with contraction ratios $\lambda_{i}$ which satisfy the separation conditions (2.2). Then we put

$$
C\left(\lambda_{1}, \ldots, \lambda_{N}\right)=\bigcap_{n=0}^{\infty} C_{n}, \quad C_{n}=\bigcup_{j=1}^{N} \sigma_{j}\left(C_{n-1}\right)
$$

and call $C$ self-similar fractal set of Cantor type.

We denote the original Cantor set by $C\left(\frac{1}{3}, \frac{1}{3}\right)$. In the case where $\lambda_{1}=\cdots=\lambda_{N}=$ $1 / M(N \leq M)$ we have the Hausdorff dimension

$$
D=\log N / \log M \text {. }
$$

We give the graph of the fractal set in the case of $C\left(\frac{1}{3}, \frac{1}{9}, \frac{1}{9}\right)$; see Fig. 2 .

$C_{0}$

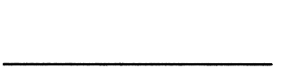

$C_{1}$

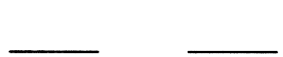

Fig. 1. The Cantor set $C\left(\frac{1}{3}, \frac{1}{3}\right)$.

$C_{0}$

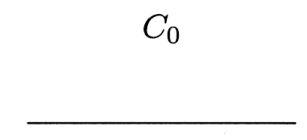

$C_{1}$

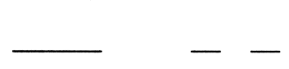

$C_{2}$

$C_{2}$

Fig. 2. Self-similar fractal set $C\left(\frac{1}{3}, \frac{1}{9}, \frac{1}{9}\right)$ of Cantor type.

3. The Hausdorff measure and integration on a fractal set. We are going to deal with the integral calculus on a fractal set. First we prepare the Hausdorff measure. Next we give the integral calculus with respect to that measure.

A collection of compact sets $\left\{U_{1}, U_{2}, \ldots, U_{m}\right\}$ is called a $\rho$-covering of $X$ if the diameter satisfies $d\left(U_{i}\right) \leq \rho(i=1, \ldots, m)$. We make the following definition $[3,5]$ :

Definition 3 ( $k$-dimensional Hausdorff measure). The $k$-dimensional Hausdorff measure is defined by

$$
\mu^{k}(X)=\lim _{\rho \rightarrow 0} \mu_{\rho}^{k}(X),
$$

where

$$
\mu_{\rho}^{k}(X) \equiv \inf \left\{\sum_{i=1}^{n} d_{i}^{k} \mid 0<d_{i} \leq \rho, X \subseteq \bigcup_{i=1}^{n} U_{i}\right\} \quad\left(d_{i}=d\left(U_{i}\right)\right)
$$

We notice the following 
Proposition 1 (Hausdorff-Besicovitch Theorem) [2, 3]. There exists a unique D $(D \geq 0)$ such that

$$
\left\{\begin{array}{l}
\mu^{k}(X)=\infty(k<D) \\
\mu^{k}(X)=0(k>D)
\end{array}\right.
$$

In the case $D=0$, we take only the latter condition.

We call $D$ the Hausdorff dimension of $X$ and $\mu^{D}$ the Hausdorff measure, respectively.

Some basic properties of the Hausdorff measure.

(1) $\mu^{D}$ is a $\sigma$-additive measure [2]:

$$
\mu^{D}\left(\sum_{j=1}^{\infty} A_{j}\right)=\sum_{j=1}^{\infty} \mu^{D}\left(A_{j}\right)
$$

for measurable sets $A_{j}(j=1,2, \ldots, n)$ with $A_{j} \cap A_{k}=\emptyset(j \neq k)$.

(2) For a measurable set $A$, we have

$$
\mu^{D}(A)=\sum_{j=1}^{N} \mu^{D}\left(\sigma_{j}(A)\right)
$$

Hence we can express the measure in terms of the so-called mass distribution [2]. Here we notice the following important fact: $\mu^{D}(K)>0$ [3]. After normalization $\mu^{D}(K)=1$, we have

$$
\mu^{D}\left(K_{j_{n} \cdots j_{1}}\right)=\lambda_{j_{n}}^{D} \cdots \lambda_{j_{1}}^{D}
$$

where

$$
K_{j_{n} \cdots j_{1}}=\sigma_{j_{n}} \circ \cdots \circ \sigma_{j_{1}}(K) .
$$

In the case of $n=1$, we arrive at the formula (2.4).

(3) The Borel algebra is generated by $\left\{K_{j_{n} \ldots j_{1}}\right\}$.

We can develop a theory of integral with respect to this measure.

4. Representations of Cuntz algebras. We introduce representations of the Cuntz algebras on the Hilbert space $L^{2}\left(K, d \mu^{D}\right)$.

The Cuntz algebra $\mathcal{O}(N)$ is a $C^{*}$-algebra with generators $\left\{S_{j}\right\}(j=1,2, . ., N)$ and the following commutation relations $[1,7]$ :

$$
\text { (1) } S_{j}^{*} S_{j}=1(j=1,2, . ., N) \text {, }
$$

(2) $\sum S_{j} S_{j}^{*}=1$.

These commutation relations give an algebraic description of the division of the total space into $N$ parts. Then we can make the representation of the Cuntz algebras on fractal sets in a well known manner:

Proposition 2 (Hausdorff representations on fractal sets of flower type) [7].

(1) Let $C$ be a self-similar fractal set defined by $\left\{\sigma_{j}\right\}(j=1,2, . . N)$. Then we have the following representation $\pi: \mathcal{O}(N) \rightarrow B\left(L^{2}(C)\right.$ :

$$
\pi\left(S_{j}\right) f(x)=\left\{\begin{aligned}
J\left(\sigma_{j}\right)^{1 / 2}\left(\sigma_{j}^{-1}(x)\right) f\left(\sigma_{j}^{-1}(x)\right), & x \in \sigma_{j}(K), \\
0, & x \notin \sigma_{j}(K)(j=1,2, \ldots, N),
\end{aligned}\right.
$$




$$
\pi\left(S_{j}^{*}\right) f(x)=J\left(\sigma_{j}\right)^{-1 / 2}(x) f\left(\sigma_{j}(x)\right) \quad(j=1,2, \ldots, N),
$$

where $J\left(\sigma_{i}\right)$ is the Radon-Nikodym derivative of $\sigma_{i}$.

The representation defined above is called Hausdorff representation.

(2) Let $C_{1}$ and $C_{2}$ be two self-similar fractal sets with the same number of generators $N$. The Hausdorff representations are equivalent if and only if they have the same Kakutani-Jørgensen invariant

$$
\left\{\lambda_{j}^{D}(j=1,2, \ldots, N)\right\} .
$$

Here two Hausdorff representations: $\pi_{i}: \mathcal{O}(N) \rightarrow B\left(L^{2}\left(C_{i}\right)\right)(i=1,2)$ are unitarily equivalent if we can find a unitary operator $U: L^{2}\left(C_{1}\right) \rightarrow L^{2}\left(C_{2}\right)$ such that $\pi_{1}(S) U=$ $U \pi_{2}(S)$ holds for any $S \in \mathcal{O}(N)$.

5. The Schauder basis on a fractal set. In this section we recall the Schauder basis on the interval and introduce a basis of Schauder type on a fractal set [4] [10, 11].

We put

$$
G^{(0)}(x)=\left\{\begin{array}{cc}
x, & x \in\left[0, \frac{1}{2}\right] \\
1-x, & x \in\left(\frac{1}{2}, 1\right]
\end{array}\right.
$$

and consider self-similar mappings $\sigma_{1}(x)=\frac{1}{2} x, \sigma_{2}(x)=\frac{1}{2} x+\frac{1}{2}$. For an integer $m$, putting

$$
G_{j_{m} \cdots j_{1}}^{(0)}(x)=\left\{\begin{array}{cl}
\frac{1}{2^{\frac{1}{2} m}} G^{(0)}\left(\sigma_{j_{1}}^{-1} \circ \cdots \circ \sigma_{j_{m}}^{-1}(x)\right) & \left(x \in I_{j_{m} \cdots j_{1}}\right), \\
0 & \text { otherwise }
\end{array}\right.
$$

we can see that these functions give a linear basis of $L^{2}(I, d \mu)$. Here we notice that

$$
\left(G_{i_{m} i_{m-1} \cdots i_{1}}^{(0)}, G_{j_{m} j_{m-1} \cdots j_{1}}^{(0)}\right)=\delta_{i_{m} j_{m}} \delta_{i_{m-1} j_{m-1}} \cdots \delta_{i_{1} j_{1}} .
$$

Yet, we can see that the different $m$ and $m^{\prime}$ are not necessarily orthonormal, for example (Fig. 3):

$$
\left(G^{(0)}, G_{j_{1}}^{(0)}\right) \neq 0
$$

Proposition 3. A continuous function $f$ with $f(0)=f(1)=0$ on $I(=[0,1])$ is expanded in the Schauder basis:

$$
f(x)=a_{0} G^{(0)}(x)+\sum_{m=1}^{\infty} \sum_{j_{1}=1}^{2} \cdots \sum_{j_{m}=1}^{2} a_{j_{m} \cdots j_{1}} G_{j_{m} \cdots j_{1}}(x) .
$$

Proof. First we take a continuous function $f(x)(f(0)=f(1)=0)$. Next we consider

$$
f_{1}(x)=f(x)-a_{0} G^{(0)}(x) \quad\left(a_{0}=2 f\left(\frac{1}{2}\right)\right)
$$

on $I(=[0,1])$. Then we have $f_{1}\left(\frac{1}{2}\right)=0$. In a similar manner, we choose $a_{j_{1}}\left(j_{1}=1,2\right)$ in

$$
f_{2}(x)=f(x)-a_{0} G^{(0)}(x)-\sum_{j_{1}=1}^{2} a_{j_{1}} G_{j_{1}}^{(0)}(x),
$$

so that

$$
f_{2}\left(\frac{1}{2^{2}}\right)=f_{2}\left(\frac{3}{2^{2}}\right)=0
$$




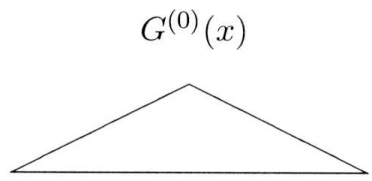

$G_{1}^{(0)}(x)$

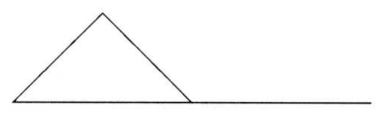

$G_{11}^{(0)}(x)$

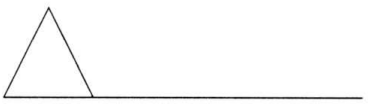

$G_{12}^{(0)}(x)$

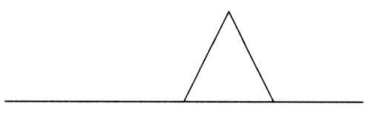

$G_{2}^{(0)}(x)$

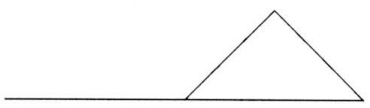

etc.

$$
G_{21}^{(0)}(x)
$$

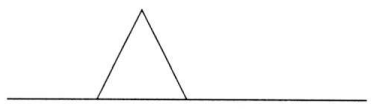

$G_{21}^{(0)}(x)$

etc.

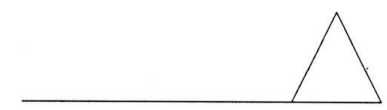

Fig. 3. A basis of Schauder type on a fractal set.

Hence we have

$$
f_{2}\left(\frac{i}{2^{2}}\right)=0 \quad(i=1, \ldots, 3) .
$$

In a similar manner, we have

$$
\begin{array}{r}
f_{n}(x)=f(x)-a_{0} G^{(0)}(x)-\sum_{m=1}^{n} \sum_{j_{1}=1}^{2} \cdots \sum_{j_{m}=1}^{2} a_{j_{m} \cdots j_{1}} G_{j_{m} \cdots j_{1}}^{(0)}(x), \\
f_{n}\left(\frac{i}{2^{n}}\right)=0 \quad\left(i=1, \ldots, 2^{n}-1\right) .
\end{array}
$$

Therefore we can see the following: For any $\epsilon>0$, there exists $n_{0}$ such that

$$
\left|f_{n}(x)\right|<\epsilon \quad\left(\forall n>n_{0}, \forall x \in I\right),
$$

which implies that $f_{n}$ converges to the constant zero function uniformly. Hence we have completed the proof. 
Next we give several kinds of bases of Schauder type.

EXAMPLE 3 (the Weierstrass basis) [2, 12].

In a similar manner to Example 1, putting

$$
W^{(0)}(x)=4 x(1-x) \quad(0 \leq x \leq 1)
$$

and choosing $\sigma_{1}, \sigma_{2}$ in Example 1, we have an orthonormal basis in $L^{2}\left(I, d \mu^{D}\right)$.

ExAmPLE 4 (the Haar-Schauder type).

Putting

$$
H^{(0)}(x)=\chi_{K}(x),
$$

$$
H_{j_{n} \cdots j_{1}}^{(0)}(x)=\left\{\begin{array}{cl}
\lambda_{j_{1}}^{-D / 2} \cdots \lambda_{j_{n}}^{-D / 2} H^{(0)}\left(\sigma_{j_{1}}^{-1} \circ \cdots \circ \sigma_{j_{n}}^{-1}(x)\right) & \text { on } K_{j_{n} \cdots j_{1}}, \\
0 & \text { otherwise }
\end{array}\right.
$$

we have a linear basis in $L^{2}\left(K, d \mu^{D}\right)$ which satisfies $(5.18)$.

EXAMPLE 5 (Schauder basis on a fractal set of Cantor type).

We can introduce a basis of Schauder type for a self-similar fractal set of Cantor type. First we consider a set which satisfies the division condition:

$$
I=\bigcup_{j=1}^{N} \sigma_{j}(I)
$$

We can give a Schauder basis in the following manner: First we define the basic functions:

$$
G^{(\rho)}=\left\{\begin{array}{cl}
\frac{1}{x_{\rho}-x_{\rho-1}}\left(x-x_{\rho}\right), & x \in I_{\rho-1, \rho}, \\
\frac{1}{x_{\rho}-x_{\rho+1}}\left(x-x_{\rho}\right), & x \in I_{\rho, \rho+1} \quad(\rho=1,2, \ldots, N-1), \\
0 & \text { otherwise. }
\end{array}\right.
$$

Then we can introduce the Schauder basis defining $G_{j_{n}, j_{n-1}, \ldots, j_{1}}^{(\rho)}$ by

$$
G_{j_{m} \cdots j_{1}}^{(\rho)}(x)=\left\{\begin{array}{cl}
G^{(\rho)}\left(\sigma_{j_{1}}^{-1} \circ \cdots \circ \sigma_{j_{m}}^{-1}(x)\right) & \left(x \in I_{j_{m} \cdots j_{1}}\right) \\
0 & \text { otherwise }
\end{array}\right.
$$

we define a basis of Schauder type, where

$$
I_{j_{m}, j_{m-1}, \ldots, j_{1}}=\sigma_{j_{m}} \circ \sigma_{j_{m-1}} \ldots \circ \sigma_{j_{1}}(I) .
$$

We can prove the uniform approximation theorem of continuous functions for this case: 
Proposition 3. For any continuous function $f$ with $f(0)=f(1)$, we can approximate $f$ by $G_{j_{n}, j_{n-1}, \ldots, j_{1}}$ uniformly:

$$
f=\sum_{\rho=1}^{N-1} a_{0}^{(\rho)} G^{(\rho)}+\sum_{\rho=1}^{N-1} \sum a_{j_{n}, j_{n-1}, \ldots, j_{1}}^{(\rho)} G_{j_{n}, j_{n-1}, \ldots, j_{1}}^{(\rho)} .
$$

In a similar manner we make a basis of Schauder type on a (general) self-similar fractal set of Cantor type $C$ (Fig. 4).
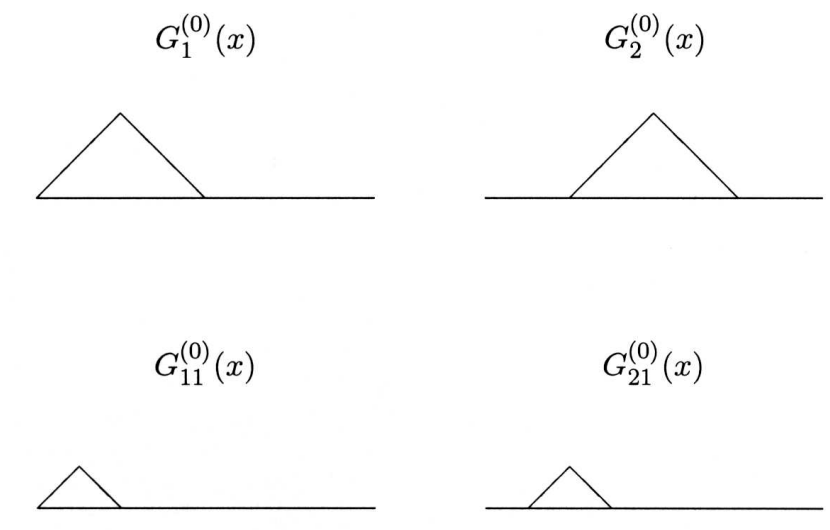

$G_{3}^{(0)}(x)$
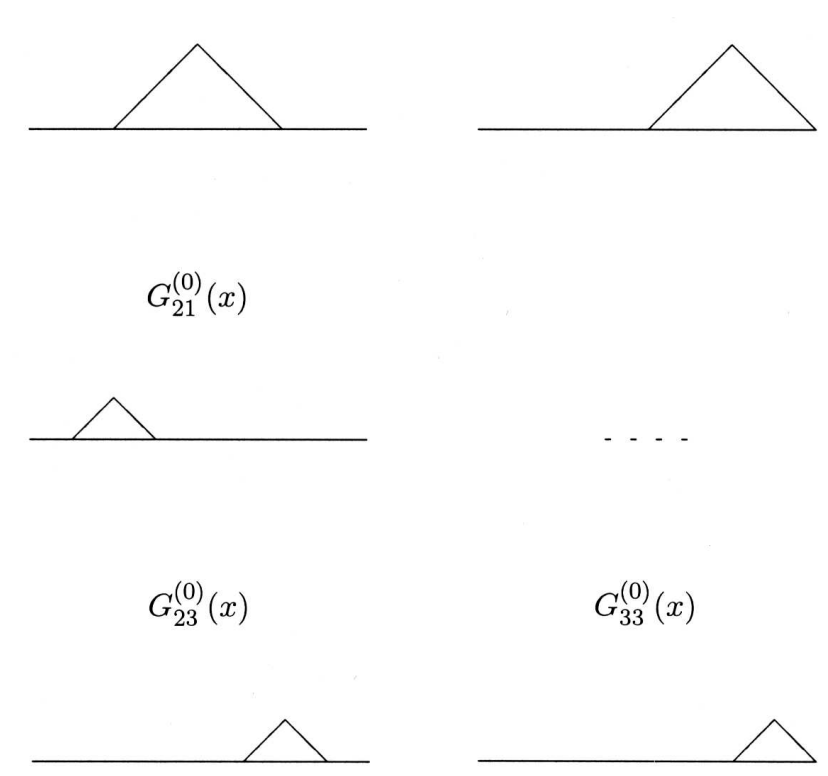

Fig. 4. A basis of Schauder type on a (general) self-similar fractal set of Cantor type.

Definition 4 (Schauder basis on a self-similar fractal set of Cantor type). Let $C$ $\left(=C\left(\lambda_{1}, \lambda_{2}, \ldots, \lambda_{N}\right)\right)$ be a fractal set of Cantor type which is generated by $\left\{\sigma_{j}: j=\right.$ $1,2, \ldots, N\}$ on the interval $I$. We set

$$
G_{j_{m} \cdots j_{1}}^{(\rho)}(x)=\left\{\begin{array}{cl}
C_{j_{m} \cdots j_{1}}^{(\rho)} G^{(\rho)}\left(\sigma_{j_{1}}^{-1} \circ \cdots \circ \sigma_{j_{m}}^{-1}(x)\right) & \left(x \in C_{j_{m} \cdots j_{1}}\right), \\
0 & \text { otherwise }
\end{array}\right.
$$

where the constants $C_{j_{m} \cdots j_{1}}^{(\rho)}$ are determined by the orthonormality conditions:

$$
\int_{C} G_{i_{m} \cdots i_{1}}^{(\rho)} G_{j_{m} \cdots j_{1}}^{\left(\rho^{\prime}\right)} d \mu^{D}=\delta_{\rho, \rho^{\prime}} \delta_{i_{m}, j_{m}} \delta_{i_{m-1}, j_{m-1}} \ldots \delta_{i_{1}, j_{1}} .
$$

We call $\left\{G^{(\rho)}, G_{j_{m} \cdots j_{1}}^{(\rho)}\right\}$ the Schauder basis on $K$. 
Then we can prove the following

TheOREM I (on Schauder basis on a self-similar fractal set of Cantor type). Let $C$ (= $C\left(\lambda_{1}, \lambda_{2}, \ldots, \lambda_{N}\right)$ be a fractal set of Cantor type. Then the system $\left\{G_{0}^{(\rho)}, G_{j_{m} \cdots j_{1}}^{(\rho)(0)}\right\}$ constitutes orthonormal basis of $L^{2}\left(C, d \mu^{D}\right)_{*}$ (cf. (7.41) below).

Proof. We take a fractal set (2.1). Next we consider the interval with the fractal structure defined by $\tilde{\sigma}_{j}: I \rightarrow I(j=1,2, \ldots, N)$ with the contraction $\operatorname{ratios}\left\{\lambda_{j}^{D}(j=1,2, \ldots, N)\right\}$. From (2.4), we have a fractal structure on $I$ satisfying the condition (5.22) and it is a fractal set of Cantor type: $I=I\left(\lambda_{1}{ }^{D}, \lambda_{2}{ }^{D}, \ldots, \lambda_{N}{ }^{D}\right)$. We construct the desired Schauder basis on the interval $I$. For an integer $m$, we put

$$
\tilde{G}_{j_{m} \cdots j_{1}}^{(\rho)}(x)=\left\{\begin{array}{cl}
C_{j_{m} \cdots j_{1}}^{(\rho)} G^{(\rho)}\left(\tilde{\sigma}_{j_{1}}^{-1} \circ \cdots \circ \tilde{\sigma}_{j_{m}}^{-1}(x)\right) & \left(x \in I_{j_{m} \cdots j_{1}}\right), \\
0 & \text { otherwise. }
\end{array}\right.
$$

Next we consider a representation of the central extension of the Cuntz algebra [1]. Setting

$$
\left\{\begin{array}{c}
S_{j}\left(G_{j_{1}, j_{2}, \ldots, j_{m}}^{(\rho)}\right)=\left(G_{j, j_{1}, j_{2}, \cdots, j_{m}}^{(\rho)}\right) \\
S_{j}^{*}\left(G_{j_{1}, j_{2}, \ldots, j_{m}}^{(\rho)}\right)=\delta_{j, j_{1}}\left(G_{j_{2}, \ldots, j_{m}}^{(\rho)}\right)
\end{array}\right.
$$

we can construct representations of the Cuntz algebra on both spaces. Comparing the Kakutani-Jørgensen invariants [4] of both representations, we have an intertwining unitary operator

$$
U: L^{2}(I, d \tilde{\mu}) \rightarrow L^{2}\left(C, d \mu^{D}\right),
$$

and this suffices to prove the assertion.

6. An orthonormal basis of Haar type on a fractal set. We are going to introduce a basis of Haar type on a fractal set. This gives an orthonormal system of the class $L^{2}\left(K, d \mu^{D}\right)$. We begin with the Haar basis on the interval $[11,12]$. We consider the following mappings:

$$
\sigma_{1}(x)=\frac{1}{2} x, \quad \sigma_{2}(x)=\frac{1}{2} x+\frac{1}{2} .
$$

Then we have the interval $I(=[0,1])$ as the self-similar fractal set. Choosing

$$
H^{(0)}(x)=1 \quad \text { on }[0,1], \quad H^{(1)}(x)=\left\{\begin{array}{cc}
1 & x \in\left[0, \frac{1}{2}\right], \\
-1 & x \in\left(\frac{1}{2}, 1\right]
\end{array}\right.
$$

we get an orthonormal system which is called Haar basis. Putting

$$
H^{(1)}{ }_{j_{m} \cdots j_{1}}(x)=\left\{\begin{array}{cl}
\frac{1}{2^{\frac{1}{2} m}} H^{(1)}\left(\sigma_{j_{1}}^{-1} \circ \cdots \circ \sigma_{j_{m}}^{-1}(x)\right) & \left(x \in C_{j_{m} \cdots j_{1}}\right), \\
0 & \text { otherwise, }
\end{array}\right.
$$

we have an orthonormal system on $L^{2}(I, d \mu)$; see Fig. 5 .

Next we proceed to an orthonormal basis of Haar type on a self-similar fractal set of general type. We can prove the following 
THEOREM I' (on an orthonormal basis of Haar type on a self-similar fractal set). Take an orthonormal system on a fractal set $K$ of Cantor type. Putting

$$
\begin{cases}H^{(0)}(x)=\chi_{K} & \left(\chi_{K}=\text { characteristic function of } K\right), \\ H^{(\rho)}(x)=\epsilon_{\rho} \chi_{K_{\rho}}-\epsilon_{\rho}^{\prime} \sum_{k=\rho+1}^{N-1} \chi_{K_{k}} & (1 \leq \rho \leq N-1),\end{cases}
$$
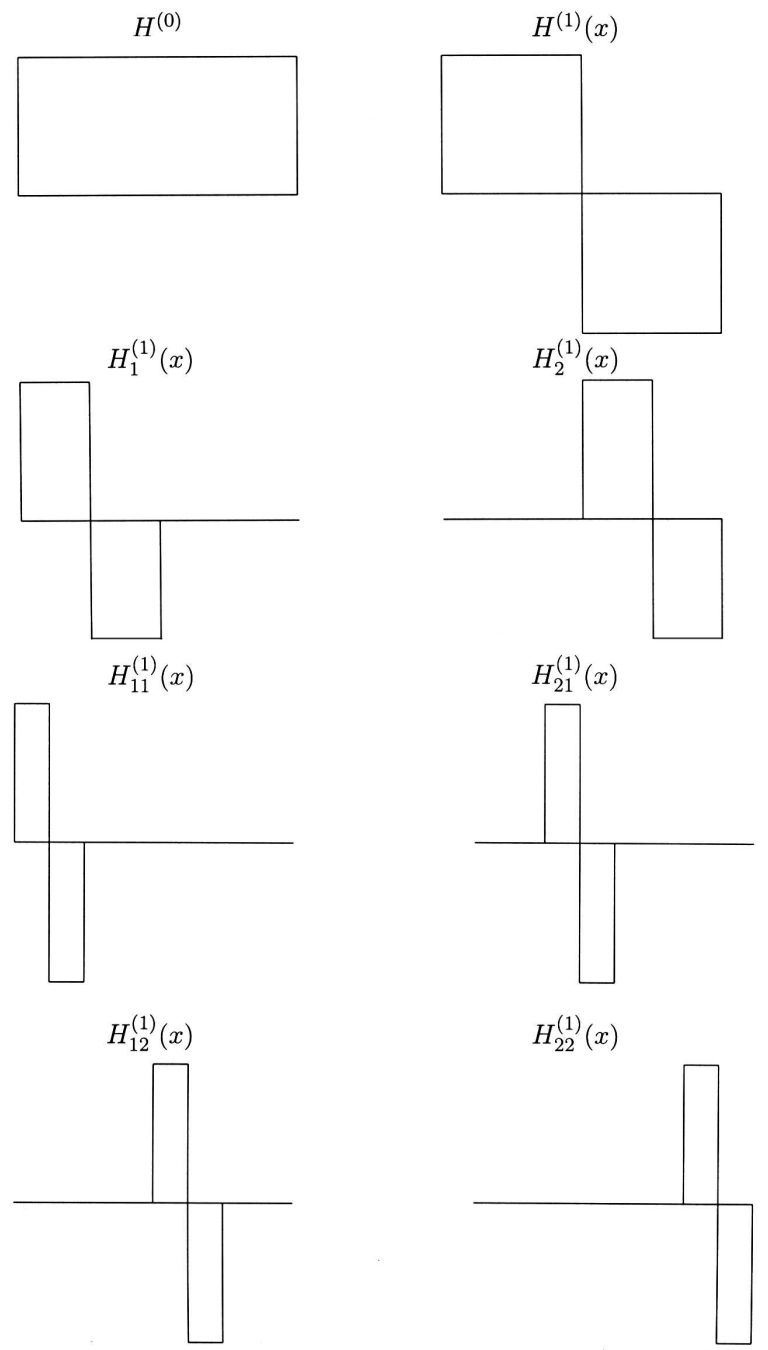

Fig. 5. An orthonormal basis of Haar type on a fractal set. 
where

$$
\epsilon_{\rho}=\sqrt{\frac{\left(1-\lambda_{1}^{D}-\cdots-\lambda_{\rho}^{D}\right)}{\lambda_{\rho}^{D}\left(1-\lambda_{1}^{D}-\cdots-\lambda_{\rho-1}^{D}\right)}},
$$

$$
\epsilon_{\rho}^{\prime}=\sqrt{\frac{\lambda_{\rho}^{D}}{\left(1-\lambda_{1}^{D}-\cdots-\lambda_{\rho}^{D}\right)\left(1-\lambda_{1}^{D}-\cdots-\lambda_{\rho-1}^{D}\right)}},
$$

we get a complete orthonormal system on $L^{2}\left(K, d \mu^{D}\right)$ :

$$
H^{(0)}(x), H^{(\rho)}(x), H_{j_{n} \cdots j_{1}}^{(\rho)}(x) \quad(\rho=1,2, \ldots, N-1),
$$

where

$$
\begin{array}{r}
H_{j_{n} \cdots j_{1}}^{(\rho)}(x)=\lambda_{j_{1}}^{-D / 2} \cdots \lambda_{j_{n}}^{-D / 2} H^{(\rho)}\left(\sigma_{j_{1}}^{-1} \circ \cdots \circ \sigma_{j_{n}}^{-1}(x)\right), \\
\left(x \in K_{j_{n} \cdots j_{1}}, \rho=1,2, \ldots, N-1\right) .
\end{array}
$$

Proof. We choose a system of functions $\left\{H_{j}^{\prime}(j=1,2, \ldots, N)\right\}$ by

$$
H_{j}^{\prime}=\chi_{K_{j}} \quad\left(K_{j}=\sigma_{j}(K)\right) .
$$

We include the constant function on $K$ :

$$
H^{(0)}=\sum_{j=1}^{N} H_{j}^{\prime}
$$

Putting

$$
H^{(1)}=\epsilon_{1} H_{1}^{\prime}+\epsilon_{1}^{\prime}\left(H_{2}^{\prime}+H_{3}^{\prime}+\cdots+H_{N}^{\prime}\right)
$$

and choosing $\epsilon_{1}$ and $\epsilon_{1}^{\prime}$ in (6.33) we can see that the orthonormality conditions hold:

$$
\left(H^{(0)}, H^{(1)}\right)=0 \quad \text { and } \quad\left(H^{(1)}, H^{(1)}\right)=1 .
$$

Next putting

$$
H^{(2)}=\epsilon_{2} H_{2}^{\prime}+\epsilon_{2}^{\prime}\left(H_{3}^{\prime}+H_{4}{ }^{\prime}+\cdots+H_{N}{ }^{\prime}\right)
$$

and choosing $\epsilon_{2}$, and $\epsilon_{2}^{\prime}$ in (6.33), we have the orthonormality conditions:

$$
\left(H^{(0)}, H^{(2)}\right)=0 \quad \text { and } \quad\left(H^{(2)}, H^{(2)}\right)=1 .
$$

Moreover, we can see that the condition

$$
\left(H^{(1)}, H^{(2)}\right)=0
$$

holds automatically by the construction. Repeating this process we can get the first $N$ basic functions:

$$
H^{(0)}, H^{(1)}, \ldots, H^{(N-1)} .
$$

Next we are going to prolongate these functions to a complete orthonormal basis: Putting (6.33), we have the orthonormality condition:

$$
\int_{K} H_{i_{n} \cdots i_{1}}^{(\rho)} H_{j_{m} \cdots j_{1}}^{\left(\rho^{\prime}\right)} d \mu^{D}=\delta_{\rho \rho^{\prime}} \delta_{n m} \delta_{i_{n} j_{n}} \cdots \delta_{i_{1} j_{1}} \quad(\rho=1,2, \ldots, N-1) .
$$


We can prove the completeness condition as follows. First we notice that every characteristic function of $K_{j}(j=1,2, \ldots, N)$ can be expressed as a linear combination of (6.33). By this we can see that the characteristic function of every generator of the Borel algebra can be written with the use of

$$
H^{(\rho)} \quad(\rho=0, \ldots, N-1), \quad H_{j_{n} \cdots j_{1}}^{(\rho)}(\rho=1,2, \ldots, N-1) .
$$

Hence we can see that the completeness condition holds for our basis as desired.

Here we can give two examples (Figs. 6 and 7).

ExAmple 6 (the Haar basis on the Cantor set) (Fig. 6).

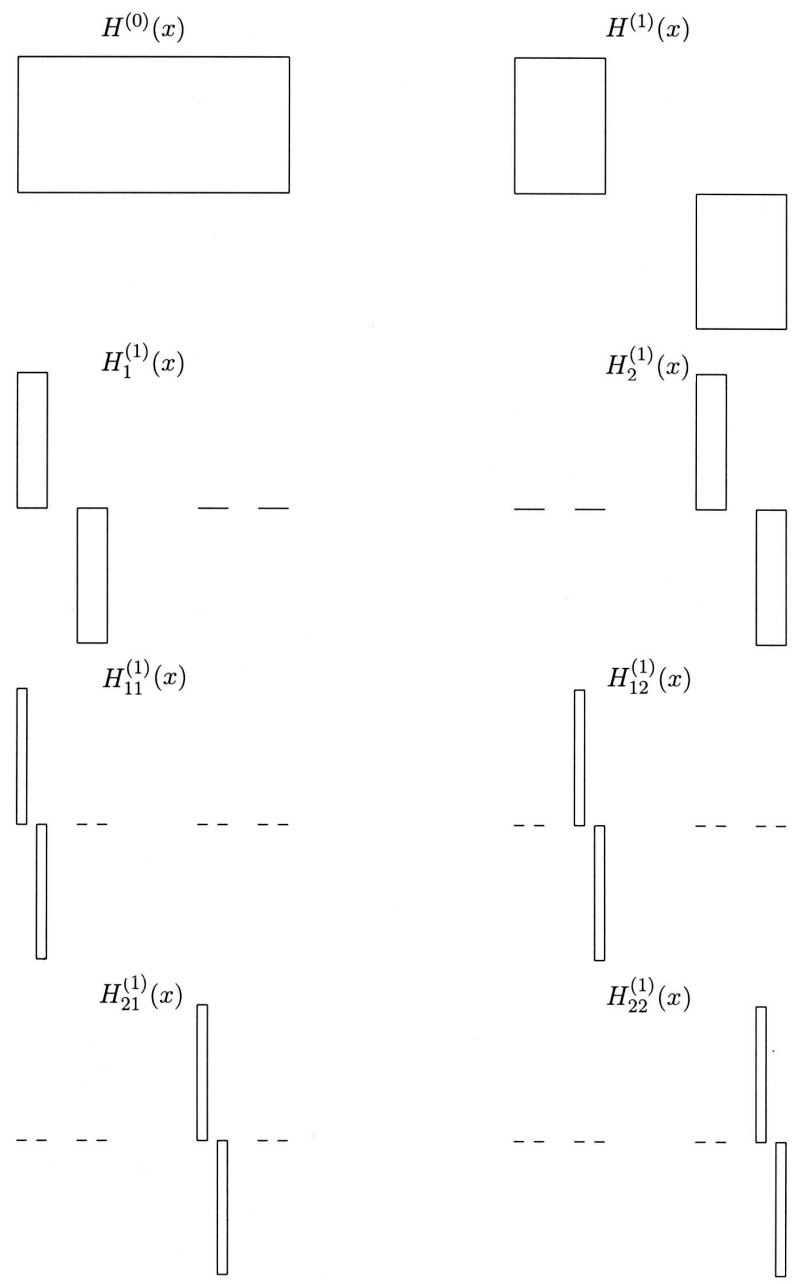

Fig. 6. The Haar basis on the Cantor set. 
EXAMPLE 7 (the Haar basis on a self-similar fractal set) (Fig. 7).
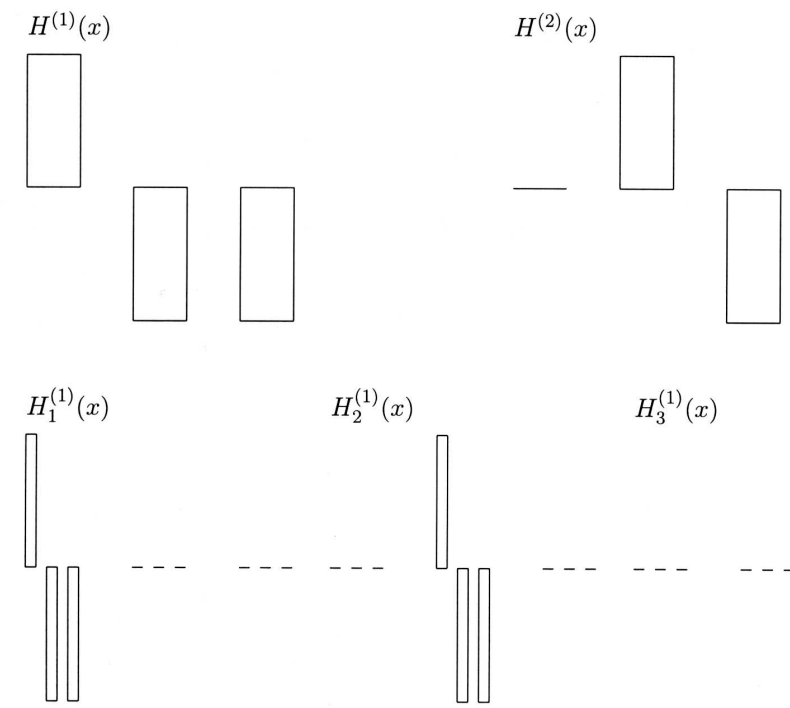

$H_{2}^{(1)}(x)$

$H_{3}^{(1)}(x)$
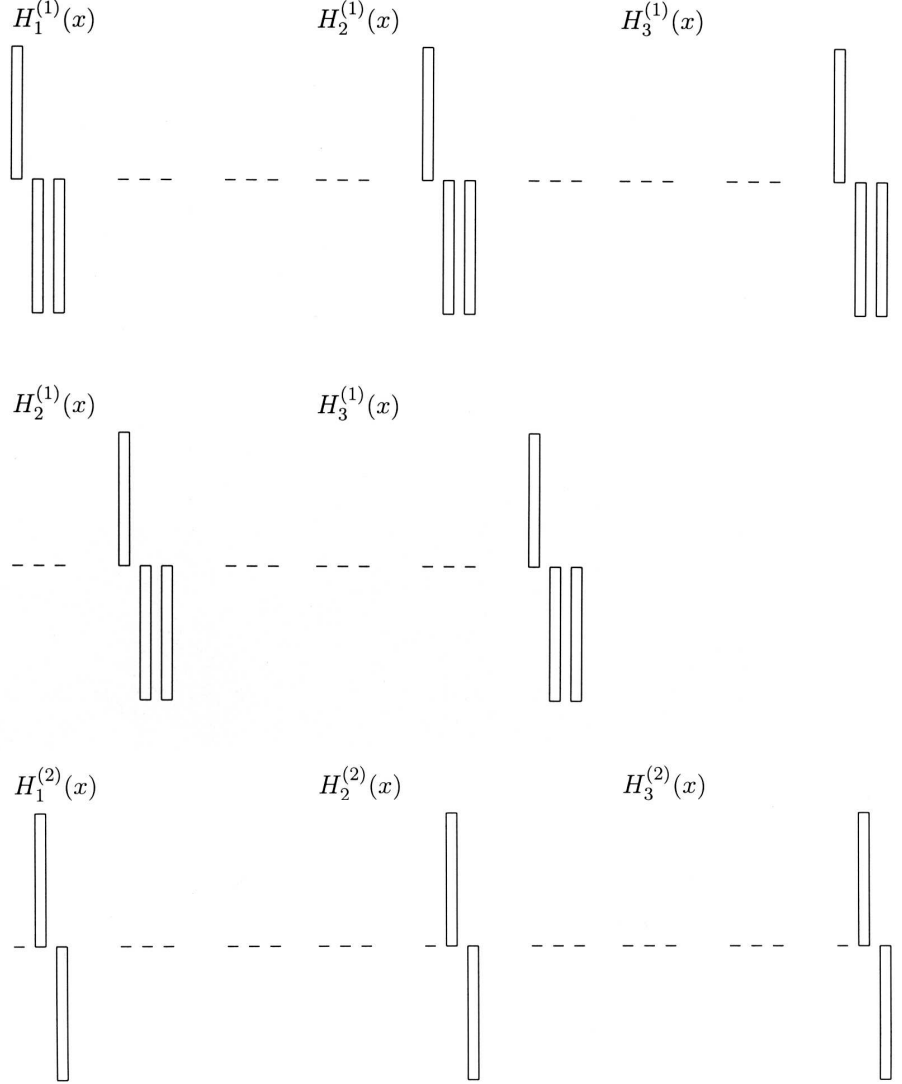

Fig. 7. The Haar basis on a self-similar fractal set.

7. A wavelet expansion of Schauder type (respectively: of Haar type) on a fractal set. In this section we give the wavelet expansion of Schauder/Haar type on a self-similar fractal set [12]. The Fourier expansion supplies an important tool in the analysis on the Euclidean space. This expansion may be regarded as the Fourier expansion on a fractal set. We can easily prove the following 
THEOREM II (Wavelet expansion theorem for the Haar basis on a self-similar fractal set). Every function of $L^{2}\left(K, d \mu^{D}\right)$ can be expressed as a linear combination of

$$
\left\{H^{(\rho)}, H_{j_{n}, \ldots, j_{1}}^{(\rho)}\right\} \text {. }
$$

Namely, for any function $f \in L^{2}\left(K, d \mu^{D}\right)$, we can find a sequence of complex numbers $\left\{a^{(\rho)}, a_{j_{n} \cdots j_{1}}^{(\rho)}\right\}$ so that

$$
\begin{gathered}
f=\sum_{\rho=1}^{N-1} a^{(\rho)} H^{(\rho)}+\sum_{n=1}^{\infty} \sum_{\rho=1}^{N-1} \sum_{j_{1}=1}^{N} \cdots \sum_{j_{n}=1}^{N} a_{j_{n} \cdots j_{1}}^{(\rho)} H_{j_{n} \cdots j_{1}}^{(\rho)}, \\
a^{(\rho)}=\left(f, H^{(\rho)}\right) \text { and } a_{j_{n} \cdots j_{1}}^{(\rho)}=\left(f, H_{j_{n} \cdots j_{1}}^{(\rho)}\right) .
\end{gathered}
$$

Next we proceed to wavelet expansions for Schauder basis on a self-similar fractal set. First we introduce a concept of degrees for Schauder basis. We call elements $G_{j_{m} \cdots j_{1}}^{(0)}(x)$ elements of degree $m$ (or $m$-forms) which we denote by $L_{m}^{2}\left(K, d \mu^{D}\right)$. Then we can see that these elements constitute an orthonormal basis of this space. Here we notice that elements of different degree are not always orthogonal, for example $G^{0}$ is not orthogonal to elements of degree $m(m>0)$. Here we introduce a new inner product defined by the orthonormalization conditions concerned with

$$
L_{m}^{2}\left(K, d \mu^{D}\right) \text { and } L_{m^{\prime}}^{2}\left(K, d \mu^{D}\right),
$$

which is called orthonormalized inner product with respect to degrees. The newly introduced inner product is denoted by $(,)_{*}$ and the space endowed with the inner product is denoted by $L_{*}^{2}\left(K, d \mu^{D}\right)$, respectively.

In a similar manner to Theorem II', we have the following

THEOREM II' (wavelet expansion theorem for the Schauder basis on a self-similar fractal set). Every function of $L_{*}^{2}\left(K, d \mu^{D}\right)$ can be expressed as a linear combination of

$$
\left\{G^{(\rho)}, G_{j_{n}, \ldots, j_{1}}^{(\rho)}\right\} .
$$

Namely, for any function $f \in L_{*}{ }^{2}\left(K, d \mu^{D}\right)$, we can find a sequence of complex numbers $\left\{a^{(\rho)}, a_{j_{n} \cdots j_{1}}^{(\rho)}\right\}$ so that

$$
\begin{gathered}
f=\sum_{\rho=1}^{N-1} a^{(\rho)} G^{(\rho)}+\sum_{\rho=1}^{N-1} \sum_{n=1}^{\infty} \sum_{j_{1}=1}^{N} \cdots \sum_{j_{n}=1}^{N} a_{j_{n} \cdots j_{1}}^{(\rho)} G_{j_{n} \cdots j_{1}}^{(\rho)}, \\
a^{(\rho)}=\left(f, G^{(\rho)}\right)_{*} \text { and } a_{j_{n} \cdots j_{1}}^{(\rho)}=\left(f, G_{j_{n} \cdots j_{1}}^{(\rho)}\right)_{*} .
\end{gathered}
$$

Examples of expansions will be given in Section 9 .

8. The $*$-products and the derivations on a fractal set. We are going to introduce a product structure on $L^{2}(K, d \mu)$ and then to define a derivation on the space. First we describe our idea on the introduction of the desired derivations. We consider the Taylor expansion of an analytic function:

$$
f=\sum_{n=0}^{\infty} \frac{a_{n}}{n !}(z-a)^{n} \quad\left(a_{n}=f^{(n)}(a)\right) .
$$


Putting $f=\left(a_{0}, a_{1}, \ldots\right)$, we have $f^{\prime}=\left(a_{1}, a_{2}, \ldots\right)$. Hence we see that the derivation is just the shift operator. Based on this fact we can get a derivation on space of sequences of numbers. We choose

$$
\Sigma=\left\{s=\left(s_{0}, s_{1}, \ldots, s_{n}, \ldots\right) \mid s_{j} \in \mathbf{C}\right\}
$$

and introduce the product structure on the space by

$$
s * t=\left(u_{0}, u_{1}, \ldots, u_{n}, \ldots\right) \quad\left(u_{n}=\sum_{j=0}^{n} s_{j} t_{n-j}\right)
$$

for two elements

$$
s=\left(s_{0}, s_{1}, \ldots, s_{n}, \ldots\right) \text { and } t=\left(t_{0}, t_{1}, \cdots, t_{n}, \ldots\right) .
$$

Then we have the following

Proposition 5. The shift operation $\sigma(\sigma: \Sigma(\mathbf{C}) \rightarrow \Sigma(\mathbf{C}))$ defines a derivation on $\Sigma$. Namely, we have

$$
\sigma(s * t)=\sigma(s) * t+s * \sigma(t) .
$$

Proof: easy and can be omitted.

Next we proceed to introducing a derivation on $L^{2}(K, d \mu)$. First we define the product structure by the following bilinear mapping:

$$
*: L^{2}(K, d \mu) \times L^{2}(K, d \mu) \rightarrow L^{2}(K, d \mu)
$$

which is determined by

$$
\left\{\begin{array}{l}
H^{(\rho)} * H^{\left(\rho^{\prime}\right)}=H^{\left(\rho^{\prime}\right)} * H^{(\rho)}=H^{(\rho)} \cdot H^{\left(\rho^{\prime}\right)}, \\
H^{(\rho)} * H_{j_{n} \cdots j_{1}}^{\left(\rho^{\prime}\right)}=\delta_{\rho \rho^{\prime}} H^{(\rho)} \cdot H_{j_{n} \cdots j_{1}}^{(\rho)} \\
H_{j_{n} \cdots j_{1}}^{(\rho)} * H^{\left(\rho^{\prime}\right)}=\delta_{\rho \rho^{\prime}} H_{j_{n} \cdots j_{1}}^{(\rho)} \cdot H^{(\rho)} \\
H_{i_{n} \cdots i_{1}}^{(\rho)} * H_{j_{n} \cdots j_{1}}^{\left(\rho^{\prime}\right)}=\delta_{\rho, \rho^{\prime}} H_{i_{n} \cdots i_{1} j_{n} \cdots j_{1}}^{(\rho)}
\end{array}\right.
$$

Then, for $f, g \in L^{2}\left(K, d \mu^{D}\right)$ :

$$
f=\sum_{\rho=0}^{N} a^{(\rho)} H^{(\rho)}+\sum_{n=1}^{\infty} \sum_{\rho=1}^{N-1} \sum_{i_{1}=1}^{N} \cdots \sum_{i_{n}=1}^{N} a_{i_{n} \cdots i_{1}}^{(\rho)} H_{i_{n} \cdots i_{1}}^{(\rho)},
$$

and

$$
g=\sum_{\rho=0}^{N} b^{(\rho)} H^{\left(\rho^{\prime}\right)}+\sum_{n=1}^{\infty} \sum_{\rho=1}^{N-1} \sum_{j_{1}=1}^{N} \cdots \sum_{j_{n}=1}^{N} b_{j_{n} \cdots j_{1}}^{\left(\rho^{\prime}\right)} H_{j_{n} \cdots j_{1}}^{\left(\rho^{\prime}\right)},
$$

and hence we have

$$
\begin{aligned}
f * g= & \sum_{\rho=0}^{N} a^{(\rho)} b^{\left(\rho^{\prime}\right)} H^{(\rho)} \cdot H^{\left(\rho^{\prime}\right)}+\sum_{n=1}^{\infty} \sum_{\rho=1}^{N-1} \sum_{i_{1}, j_{1}=1}^{N} \cdots \sum_{i_{n}, j_{n}=1}^{N} a_{i_{n} \cdots i_{1}}^{(\rho)} b_{j_{n} \cdots j_{1}}^{\left(\rho^{\prime}\right)} H_{i_{n} \cdots i_{1}}^{(\rho)} * H_{j_{n} \cdots j_{1}}^{\left(\rho^{\prime}\right)} \\
= & \sum_{\rho=0}^{N} a^{(\rho)} b^{(\rho)} H^{(\rho)} \cdot H^{\left(\rho^{\prime}\right)}+\sum_{m=1}^{\infty} \sum_{\rho=1}^{N-1} \sum_{i_{1}=1}^{N} \cdots \sum_{i_{m}=1}^{N} c_{i_{m} \cdots i_{1}}^{(\rho)} H_{i_{m} \cdots i_{1}}^{(\rho)} \\
& \left(c_{i_{m} \cdots i_{1}}^{(\rho)}=\delta_{\rho \rho^{\prime}} \sum a_{i_{n-k} \cdots i_{1}}^{(\rho)} b_{i_{m} \cdots i_{n-k+1}}^{\left(\rho^{\prime}\right)}\right),
\end{aligned}
$$


which we call the $*$-product on $L^{2}(K, d \mu)$ with respect to the basis $\{H\}$. We notice the following

Proposition 6. We have

$$
\begin{cases}(1) & \left(f_{1}+f_{2}\right) * g=f_{1} * g+f_{2} * g \text { and } f *\left(g_{1}+g_{2}\right)=f * g_{1}+f * g_{2} \\ (2) & (f * g) * h=f *(g * h) .\end{cases}
$$

Proof: easy and can be omitted.

Then we can prove the following

TheOrem III (derivation on a self-similar fractal set). Putting

$$
\left\{\begin{array}{l}
(1) \quad \delta_{i} H^{(\rho)}=0 \\
(2) \quad \delta_{i}\left(H^{(\rho)} * H_{j_{n} \cdots j_{1}}^{(\rho)}\right)=H^{(\rho)} * \delta_{i} H_{j_{n} \cdots j_{1}}^{(\rho)} \quad(i=1, \ldots, N), \\
(3) \quad \delta_{i}\left(H_{i_{n}, \ldots, i_{1}}^{(\rho)}\right)=\sum_{k=1}^{n} \delta_{i, i_{k}} H_{i_{n}, \cdots, \check{i}_{k}, \ldots, i_{1}}^{(\rho)},
\end{array}\right.
$$

where the notation $\check{i}_{k}$ means omitting the index $i_{k}$, we have the derivations on $L^{2}(K, d \mu)$ :

$$
\delta_{i}(f * g)=\delta_{i}(f) * g+f * \delta_{i}(g) \quad(i=1, \ldots, N) .
$$

Proof. First we prove the assertion for functions of the form

$$
F=\sum_{k=1}^{\infty} \sum a_{i_{k} \cdots i_{1}}^{(\rho)} H_{i_{k} \cdots i_{1}}^{(\rho)}, \quad G=\sum_{l=1}^{\infty} \sum b_{j_{l} \cdots j_{1}}^{(\rho)} H_{j_{l} \cdots j_{1}}^{(\rho)} .
$$

Then we have

$$
\begin{aligned}
& F * G=\sum_{m=1}^{\infty} \sum c_{p_{m} \cdots p_{1}}^{(\rho)} H_{p_{m} \cdots p_{1}}^{(\rho)} \\
& \left(c_{p_{m} \cdots p_{1}}^{(\rho)}=a_{1} b_{p_{m} \cdots p_{1}}+a_{p_{1}} b_{p_{m} \cdots p_{2}}+\cdots+a_{p_{m} \cdots p_{1}} b_{1}\right) .
\end{aligned}
$$

Next we introduce the derivations

$$
\begin{aligned}
& \delta_{x} F=\sum_{k=1}^{\infty} \sum_{\alpha=1}^{k} \sum a_{i_{k} \cdots i_{1}}^{(\rho)} \delta_{x, i_{\alpha}} H_{i_{k} \cdots i_{\alpha} \cdots i_{1}}^{(\rho)}, \\
& \delta_{x} G=\sum_{l=1}^{\infty} \sum_{\beta=1}^{l} \sum b_{j_{l} \cdots j_{1}}^{(\rho)} \delta_{x, j_{\beta}} H_{j_{l} \cdots \tilde{j_{\beta} \cdots j_{1}}}^{(\rho)} .
\end{aligned}
$$

Then we have

$$
\begin{aligned}
\delta_{x}(F * G) & =\sum_{m=1}^{\infty} \sum_{\alpha=1}^{m} \sum a_{p_{m} \cdots p_{k}}^{(\rho)} b_{p_{k+1} \cdots p_{1}}^{(\rho)} \delta_{x, p_{\alpha}} H_{p_{m} \cdots p_{\alpha} \cdots p_{k+1} p_{k} \cdots p_{1}}^{(\rho)} \\
& +\sum_{m=1}^{\infty} \sum_{\alpha=1}^{m} \sum a_{p_{m} \cdots p_{k}}^{(\rho)} b_{p_{k+1} \cdots p_{1}}^{(\rho)} \delta_{x, p_{\alpha}} H_{p_{m} \cdots p_{k+1} p_{k} \cdots p_{\alpha} \cdots p_{1}}^{(\rho)} .
\end{aligned}
$$


On the other hand, we get

$$
\begin{aligned}
\left(\delta_{x} F\right) * G & =\left(\sum_{k=1}^{\infty} \sum_{\alpha=1}^{k} \sum a_{i_{k} \cdots i_{1}}^{(\rho)} \delta_{x, i_{\alpha}} H_{i_{k} \cdots i_{\alpha} \cdots i_{1}}^{(\rho)}\right) *\left(\sum_{l=1}^{\infty} \sum b_{j_{l} \cdots j_{1}}^{\left(\rho^{\prime}\right)} H_{j_{l} \cdots j_{1}}^{\left(\rho^{\prime}\right)}\right) \\
& =\sum_{k=1}^{\infty} \sum_{\alpha=1}^{k} \sum_{l=1}^{\infty} \sum \sum a_{i_{k} \cdots i_{1}}^{(\rho)} b_{j_{l} \cdots j_{1}}^{(\rho)} \delta_{x, i_{\alpha}} H_{i_{k} \cdots i_{\alpha} \cdots i_{1} j_{l} \cdots j_{1}}^{(\rho)}, \\
F *\left(\delta_{x} G\right)= & \left(\sum \sum_{k=1}^{\infty} \sum a_{i_{k} \cdots i_{1}}^{(\rho)} H_{i_{k} \cdots i_{1}}^{(\rho)}\right) *\left(\sum_{l=1}^{\infty} \sum_{\beta=1}^{l} \sum b_{j_{l} \cdots j_{1}}^{\left(\rho^{\prime}\right)} \delta_{x, j_{\beta}} H_{j_{l} \cdots j_{\beta} \cdots j_{1}}^{\left(\rho^{\prime}\right)}\right) \\
= & \sum_{k=1}^{\infty} \sum_{\beta=1}^{l} \sum_{l=1}^{\infty} \sum \sum a_{i_{k} \cdots i_{1}}^{(\rho)} b_{j_{l} \cdots j_{1}}^{\left(\rho^{\prime}\right)} \delta_{x, j_{\beta}} H_{i_{k} \cdots i_{1} j_{l} \cdots j_{\beta} \cdots j_{1}}^{(\rho)} .
\end{aligned}
$$

which proves the assertion.

Next we prove the assertion of Theorem II in the general case. Choosing

$$
f=f_{0}+F, \quad g=g_{0}+G \quad\left(f_{0}=\sum_{\rho=0}^{N-1} a^{(\rho)} H^{(\rho)}, \quad g_{0}=\sum_{\rho^{\prime}=0}^{N-1} b^{\left(\rho^{\prime}\right)} H^{\left(\rho^{\prime}\right)}\right),
$$

and using $\delta_{x}\left(f_{0}\right)=0$, we easily prove the assertion, as desired.

REMARK 1. We can define a derivation for a linear basis which is not necessarily orthonormal, for example, for a Schauder basis.

9. The Taylor expansion theorem on a fractal set. In this section we give the Taylor expansion theorem on a self-similar fractal set including several examples of Taylor expansions. First we take an orthonormal basis $\left\{H^{(\rho)}, H_{j_{n} \cdots j_{1}}^{(\rho)}\right\}$ of Haar type and state the Taylor expansion. We introduce a special class of functions:

DEFINITION 5 (symmetric functions).

(1) A function $f$ of $L^{2}(K, d \mu)$ is called symmetric if

$$
f=\sum a_{i_{m} \cdots i_{1}}^{(\rho)} H_{i_{m} \cdots i_{1}}^{(\rho)}, \quad a_{i_{m} \cdots i_{1}}^{(\rho)}=a_{\tau\left(i_{m}\right) \tau\left(i_{m-1}\right) \cdots \tau\left(i_{1}\right)}^{(\rho)},
$$

where $\tau$ is a permutation of $N$ symbols.

(2) A function $f$ of $L^{2}(K, d \mu)$ is called of one variable type, if

$$
a_{i_{m} \cdots i_{1}}^{(\rho)}=a_{j_{m} \cdots j_{1}}^{(\rho)}
$$

for any pair of $\left(i_{m} \cdots i_{1}\right)$ and $\left(j_{m} \cdots j_{1}\right)$. In that case we have

$$
f=\sum a_{n}^{(\rho)} R_{n}^{(\rho)}, \quad R_{n}^{(\rho)}=\sum R_{i_{m} \cdots i_{1}}^{(\rho)} .
$$

Then we can prove the following 
THEOREM IV (Taylor expansion theorem). A symmetric function $f$ of $L^{2}(K, d \mu)$ has the following Taylor expansion:

$$
\begin{gathered}
f=\sum a_{i_{n} \cdots i_{1}}^{(\rho)} H_{i_{n} \cdots i_{1}}^{(\rho)}, \\
a_{i_{n} \cdots i_{1}}=\frac{1}{n_{1} ! n_{2} ! \ldots n_{N} !} \delta_{1}^{m_{1}} \ldots \delta_{N}^{n_{N}} f\left(H^{(\rho)}\right) \quad\left(n_{1}+n_{2}+\ldots+n_{N}=n\right),
\end{gathered}
$$

where $f\left(H^{(\rho)}\right)=\left(f, H^{(\rho)}\right)$.

Proof. We take an element $f$ of $L^{2}\left(K, d \mu^{D}\right)$ with symmetric coefficients. Then we can see that

$$
\begin{array}{ccc}
\left(\delta_{i} f, H^{(\rho)}\right) & = & a_{i}, \\
\left(\delta_{i} \delta_{j} f, H_{(\rho)}\right) & = & a_{i j}+a_{j i}, \\
\cdots & & \cdots \\
\left(\delta_{i_{m}} \cdots \delta_{i_{1}} f, H^{(\rho)}\right) & = & \sum_{\tau \in G_{m}} a_{\tau\left(i_{m}\right) \cdots \tau\left(i_{1}\right)},
\end{array}
$$

where $G_{m}$ is the $m$-th symmetric group.

Hence, for a symmetric function, we have

$$
a_{i_{m} \cdots i_{1}}=\frac{1}{n_{1} ! n_{2} ! \ldots n_{N} !} \delta_{1}^{m_{1}} \cdots \delta_{N}^{n_{N}} f\left(H^{(\rho)}\right) .
$$

We give several examples.

ExAmPLE 8 (Haar functions) [12]. The Haar basis constitutes a complete orthonormal basis of $L^{2}(I, d \mu)$ and every function can be expanded into the Taylor expansion in our sense.

Next we give the Taylor expansion for a non-orthonormal basis. We take the Schauder basis (5.26). To state the Taylor expansion theorem, we prepare the following notation: For

$$
f=\sum a_{j_{n} \cdots j_{1}} G_{j_{n} \cdots j_{1}}
$$

we define

$$
\left\langle f, G_{j_{n} \cdots j_{1}}\right\rangle:=a_{j_{n} \cdots j_{1}} .
$$

Following the discussion in the case of an orthonormal basis, we can prove the Taylor expansion theorem:

THEOREM IV' (Taylor expansion for Schauder basis). A symmetric function $f$ of $L^{2}(K, d \mu)$ has the following Taylor expansion:

$$
\begin{aligned}
f & =\sum a_{j_{n} \cdots j_{1}}^{(\rho)} G_{j_{n} \cdots j_{1}}^{(\rho)}, \\
a_{j_{n} \cdots j_{1}} & =\frac{1}{n_{1} ! n_{2} ! \ldots n_{N} !} \delta_{1}^{m_{1}} \cdots \delta_{N}^{n_{N}} f\left(H^{(\rho)}\right),
\end{aligned}
$$

where $f\left(G^{(\rho)}\right)=\left\langle f, G^{(\rho)}\right\rangle$. 
EXAMPLE 9 (Takagi function) $[2,10,11]$. We consider a fractal structure on $I$ which is defined by the mappings (6.31) and we choose the function $G_{0}$. The Takagi function is given by:

$$
T(x)=\sum_{n=0}^{\infty}\left(\frac{1}{2}\right)^{n} G_{n}(x),
$$

where

$$
G_{n}(x)=\sum G_{0}\left(\sigma_{j_{1}}^{-1} \circ \cdots \circ \sigma_{j_{n}}^{-1}(x)\right) .
$$

Hence we can see that the function is of one variable type and we have

$$
\delta^{n} T\left(G_{0}\right)=n !\left(\frac{1}{2}\right)^{n} .
$$

EXAMPLE 10 (Schauder expansion) [7]. We notice that we can regard the Schauder expansion as the Taylor expansion. We can expand any continuous function $f$ on $I$ (= $[0,1])$ with $f(0)=f(1)=0$ as the Schauder expansion:

$$
f(x)=\sum_{n=1}^{\infty} a_{j_{n} \cdots j_{1}} G_{j_{n} \cdots j_{1}}(x),
$$

where

$$
G_{j_{n} \cdots j_{1}}(x)=G_{0}\left(\sigma_{j_{1}}^{-1} \circ \cdots \circ \sigma_{j_{n}}^{-1}(x)\right) .
$$

Then, in the case where $f$ is symmetric, we can see that

$$
a_{j_{n} \cdots j_{1}}=\frac{1}{n !} \delta_{j_{n}} \cdots \delta_{j_{1}} f\left(G_{0}\right) .
$$

EXAMPLE 11 (Weierstrass function) [2, 12]. We consider the fractal set $I$ defined by

$$
\sigma_{1}(x)=\frac{1}{2}(1+\sqrt{x+1}), \quad \sigma_{2}(x)=\frac{1}{2}(1-\sqrt{x+1}) .
$$

We put

$$
W_{0}(x)=4 x(1-x),
$$

and

$$
W_{j_{n} \cdots j_{1}}(x)=W_{0}\left(\sigma_{j_{1}}^{-1} \circ \cdots \circ \sigma_{j_{n}}^{-1}(x)\right) .
$$

The Weierstrass function is given by

$$
W(x)=\sum_{n=0}^{\infty}\left(\frac{1}{2}\right)^{n} W_{n}(x),
$$

where $W_{n}$ are symmetrizations of $W_{j_{n} \cdots j_{1}}$. Hence we can see that

$$
\delta^{n} W\left(W_{0}\right)=n !\left(\frac{1}{2}\right)^{n} .
$$

10. Basic functions on a self-similar fractal set. We are going to introduce basic functions, i.e., exponential, trigonometrical, and geometric functions on a self-similar fractal set and derive differential equations of them. Then we give a close relationship between these functions and the corresponding functions on the Euclidean space. We 
can show that the usual exponential, trigonometrical, and geometric functions can be embedded into the fractal basic functions.

We take a self-similar fractal set $K$ and choose a basis of Schauder type

$$
\left\{G_{0}^{(\rho)}, G_{j_{m}, j_{m-1}, \ldots, j_{1}}^{(\rho)}\right\} .
$$

We make the symmetrization of these functions with respect to a fixed degree $m$ and obtain functions of one variable: $\left\{R^{0}, R^{m}\right\}$. Then we can introduce basic functions in terms of the Taylor expansion theorem:

$$
\left\{\begin{array}{l}
\text { (1) } \frac{1}{1-R}=R^{0}+R^{1}+R^{2}+\ldots+R^{n}+\ldots \\
\text { (2) } \sin R=R^{1}-\frac{1}{3 !} R^{3}+\frac{1}{5 !} R^{5}-\ldots+(-1)^{n} \frac{1}{2 n-1 !} R^{2 n-1}+\ldots, \\
\text { (3) } \cos R=R^{0}-\frac{1}{2 !} R^{2}+\frac{1}{4 !} R^{4}-\ldots+(-1)^{n} \frac{1}{2 n-1 !} R^{2 n}+\ldots \\
\text { (4) } \mathrm{e}^{R}=R^{0}+\frac{1}{1 !} R^{1}+\frac{1}{2 !} R^{2}+\ldots+\frac{1}{n !} R^{n}+\ldots
\end{array}\right.
$$

Here we understand the series as formal series and the convergences are not considered. Then we can see that they satisfy the differential equations as in the case of the corresponding basic functions: Putting

$$
\delta=\frac{1}{N} \sum_{\rho=1}^{N-1} \delta^{(\rho)}
$$

we have

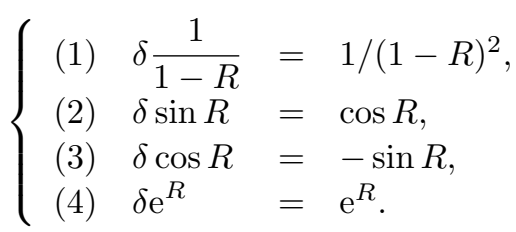

In the final section we shall show that these basic functions can describe fluctuations which can be observed in the nature.

In turn we give an intimate relationship between the usual basic functions and fractal basic functions mentioned above. Namely, we can prove the following embedding theorem:

THEOREM V (on the fluctuation mapping). Let $K$ be a self-similar fractal set and let (10.59) be a Schauder basis on it. Let $\left\{R^{0}, R^{m}\right\}(=\mathbb{C}[R])$ be the one-dimensional reduction. Then we can get the following embedding which preserves the derivation structure: Putting $\iota(x)=R$, we have a ring isomorphism satisfying the commutative diagram

$$
\begin{array}{cr}
\iota: \mathbb{C}[x] \rightarrow & \mathbb{C}[R] \\
\frac{d}{d x} \downarrow & \downarrow \delta \\
\iota: \mathbb{C}[x] \rightarrow & \mathbb{C}[R]
\end{array}
$$


Definition 6 (fluctuation mapping). We call the embedding in Theorem $\mathrm{V}$ the fluctuation mapping.

REMARK 2. Here we have to notice the following fact: Our embedding $\iota(x)=R$ should be understood as a noncommutative isomorphism between the function spaces $\mathbb{C}[x]$ and $\mathbb{C}[R]$. In the case where the fractal set $K$ is a boundary of some domain $D$ in the complex plane $\mathbb{C}$, we can see that the derivation on $K$ cannot be obtained as the restriction of the usual derivation:

$$
\left.\frac{d}{d z}\right|_{K} \neq \delta
$$

This will be discussed in the next section.

We conclude this section with a comment on the relationship between the Schauder expansion and the discrete Laplace operator. First we give

DEFinition 7 (discrete Laplace operator on a self-similar fractal set). Let $K$ be a selfsimilar fractal set which is defined by a system of contractions $\left\{\sigma_{j}: j=1,2, \ldots, N\right\}$. Then we define the Laplace operator

$$
\Delta: L^{2}\left(K, d \mu^{D}\right) \rightarrow L^{2}\left(K, d \mu^{D}\right)
$$

of the fractal set by

$$
\Delta f(p)=\sum_{j=1}^{N} f\left(\sigma_{j}(p)\right)-f(p) .
$$

We notice that it can be expressed in terms of the derivations:

$$
\Delta f(p)=\sum_{j=1}^{N} \delta_{j} f(p)-f(p) .
$$

Then we may expect to prove the following formula: For a function $f$ which is the restriction of a continuous function on the ambient space, we have

$$
f=\sum_{\rho=1}^{N-1} a^{(\rho)} R^{(\rho)}+\sum_{\rho=1}^{N-1} \sum_{n=1}^{\infty} \sum_{j_{1}=1}^{N} \cdots \sum_{j_{n}=1}^{N} a_{j_{n} \cdots j_{1}}^{(\rho)} R_{j_{n} \cdots j_{1}}^{(\rho)} .
$$

In consequence,

$$
a_{j_{n} \cdots j_{1}}^{(\rho)}=<\Delta f, R_{j_{n} \cdots j_{1}}^{(\rho)}>.
$$

REMARK 3. In the case where $K=I, I$ being the interval with the fractal structure defined by (5.26), we can see that the above mentioned formula holds [12].

ExAmple 12 (Takagi function). The Takagi function can be obtained by solving the equation

$$
T\left(G^{(0)}(x)\right)=2 T(x)+G^{(0)}(x)
$$

Putting $T(x)=\sum a_{n} R^{n}$, we can obtain the solution by solving the equations

$$
a_{n}=\left\langle\Delta f, R^{n}\right\rangle\left(=\frac{1}{2^{n-1}}\right) .
$$

EXAMPLE 13 (Weierstrass function). The Weierstrass functions can be treated in a completely similar manner. In the next section we shall consider the dynamical system for a 
self-similar fractal set and the corresponding generating functions. These functions can be treated analogously; see [12].

11. Dynamical systems of a fractal set and differential equations for their generating functions. In this section we introduce the dynamical system for a selfsimilar fractal set and show that the corresponding generating functions can be expressed in terms of the generators of the one-dimensional part. We take a self-similar fractal set $K$ which is defined by contractions $\left\{\sigma_{j}: j=1,2, \ldots, N\right\}$. Then with $\Phi: K \rightarrow K$ given by

$$
\Phi=\sigma_{j}^{-1} \text { on } K_{j}
$$

we can define the dynamical system $\left\{x_{n}\right\}$ with an initial value $x_{0} \in K$ by

$$
x_{n}=\Phi\left(x_{n-1}\right)(n=1,2, . .) .
$$

Then we can see that the dynamical system becomes a chaotic dynamical system. Moreover,

(1) We have a dense subset of periodic orbits and

(2) There exists a dense orbit and

(3) The behaviour of the dynamical system is sensitive to the choice of initial values.

Next we consider the generating function of the dyamical system with respect to the base function $g \in L^{2}\left(K, d \mu^{D}\right)$ :

$$
\left\{\begin{array}{l}
G(g: x, t)=\sum_{n=0}^{\infty} t^{n} g\left(\Phi^{n}(x)\right), \\
\hat{G}(g: x, t)=\sum_{n=0}^{\infty} \frac{t^{n}}{n !} g\left(\Phi^{n}(x)\right) .
\end{array}\right.
$$

In the case where $g(x)=G_{0}(x)$, we call $g$ the generating function of the Schauder basis. In this case we can see that

$$
\left\{\begin{array}{l}
G\left(G_{0}: x, t\right)=\sum_{n=0}^{\infty} t^{n} \Phi^{n}(x), \\
\hat{G}\left(G_{0}: x, t\right)=\sum_{n=0}^{\infty} \frac{t^{n}}{n !} \Phi^{n}(x) .
\end{array}\right.
$$

We arrive at

Proposition 7. We have

(1) $\Phi^{n}(x)=R^{n}(x)$.

(2) The shift operation $\sigma$ given by $\sigma g\left(\Phi^{n}(x)\right)=g\left(\Phi^{n+1}(x)\right)$ satisfies the relation

$$
\sigma^{*} G(z, t)=\frac{1}{t}(G(z: x, t)-\Phi(z)) .
$$


(3) In the case of a generating function of the Schauder basis,

$$
\delta(\hat{G}(z, t))=t \hat{G}(z, t) .
$$

Proof.

(1) Choosing the base function $\Phi(z)$, we get

$$
\begin{aligned}
\Phi^{(1)}(z) & =\Phi\left(\sigma^{-1}(z)\right) \text { on } K_{j}, \\
\Phi^{(2)}(z) & =\Phi\left(\sigma_{j_{1}} \sigma_{j_{2}}\right) \text { on } K_{j_{2}, j_{1}}, \\
\ldots \ldots & \\
\Phi^{(n)}(z) & =\Phi\left(\sigma_{j_{1}} \sigma_{j_{2}} \ldots \sigma_{j_{n}}\right) \text { on } K_{j_{n}, \ldots, j_{2}, j_{1}} .
\end{aligned}
$$

Hence the assertion follows.

(2) We calculate:

$$
\begin{aligned}
G(\Phi: x, t) & \left.=\sum_{n=0}^{\infty} t^{n} \Phi\left(\Phi^{n}(x)\right)=\sum_{n=0}^{\infty} t^{n} \Phi^{n+1}(x)\right) \\
& \left.\left.=\frac{1}{t} \sum_{n=0}^{\infty} t^{n+1} \Phi^{n+1}(x)\right)=\frac{1}{t} \sum_{n=1}^{\infty} t^{n} \Phi^{n}(x)\right) \\
& =\frac{1}{t}(G(z: x, t)-\Phi(z)) .
\end{aligned}
$$

(3) This is a well known property of generating functions.

12. Realization of fluctuations by fluctuation mappings. In the final section we are going to show that fluctuations observed in the nature can be realized as the image of fluctuation mappings of smooth functions without fluctuations.

Several physists have discussed the appearance of background fluctuations in the nature. Recently Nottale [8] has introduced the concept of the scale symmetry and tried to understand the hierarchy structure of the universe and the fluctuations. One of the authors of this paper has discussed the "background fluctuations of the nature" in terms of Hurwitz pairs. He has introduced additional dimensions for fluctuations and made a trial of including fluctuations in mathematics.

First we notice that important functions, for example, Takagi and Weierstrass functions are nothing but the generating functions of the Schauder basis (see (9.48) and (9.57)). Hence we may expect getting functions with big fluctuations as images of holomorphic functions without fluctuations by the fluctuation mappings. Here we shall show that we can realize in such a way fluctuations observed in nature.

We consider a real analytic function

$$
f(z)=\sum_{n=0}^{\infty} c_{n} z^{n} \quad(|z| \leq 1) .
$$

Choosing a fractal set $K$ with a Schauder basis, making its one-dimensional part $\left\{R^{n}\right\}$ and using the mapping $\iota: \mathbb{C}[z] \rightarrow \mathbb{C}[R]$, we have 


$$
\iota(f)=\sum_{n=0}^{\infty} c_{n} R^{n},
$$

and then we can realize fluctuations by a suitable choice of $\left\{c_{n}\right\}$.

Here we are concerned with the following two kinds of wavelets:

(1) Wavelet expansions of Weierstrass functions type

We consider the dynamical system on the unit circle $S=\partial \mathbf{D}$ defined by

$$
\Phi(\theta)=b \theta,
$$

where $b$ is an integer, $b>1$. This dynamical system can be realized as the boundary values of the holomorphic dynamical system defined by $\tilde{\Phi}(z)=z^{b}$ :

$$
z, z^{b}, z^{b^{2}}, \ldots, z^{b^{n}}, \ldots
$$

In that case the generating function can be written as

$$
\tilde{G}(z, t: z)=\sum_{i=1}^{\infty} t^{n} z^{b^{n}} .
$$

Taking the boundary value of the function and restricting its real part we get the Weierstrass function. We notice that the derivation is not the restriction to the boundary. Namely, we have the formula

$$
\delta\left(z^{b^{n}}\right)=n z^{b^{n-1}} .
$$

Choosing coefficients $\left\{a^{(\rho)}, a_{j_{n} \cdots j_{1}}^{(\rho)}\right\}$, we can realize several fluctuations, for instance in music and geography.

(2) Wavelet expansions of exponential logistic type

Finally we are concerned with fluctuations which can be obtained by the dynamical system of the logistic type on the unit circle $S=\partial \mathbf{D}$ :

$$
\Phi(\theta)=a \theta(2 \pi-\theta),
$$

where $\mathbf{D}$ is the unit disc and $a$ is a constant. We can realize it as the boundary values of the following non-holomorphic dynamical system on $\mathbf{D}$ :

$$
\tilde{\Phi}^{n}(z)=r^{n} e^{i \Phi^{n}(\theta)} .
$$

We notice that this is not realized as the boundary values of a holomorphic dynamical system on D. Choosing coefficients $\left\{a^{(\rho)}, a_{j_{n} \ldots j_{1}}^{(\rho)}\right\}$, we can realize several fluctuations in the behaviours of the stock prices, neuron systems and music. For examples, we refer to our paper [9].

\section{References}

[1] J. Cuntz, Simple $C^{*}$-algebras generated by isometries, Comm. Math. Phys. 57 (1977), 173185.

[2] K. Falconer, Fractal Geometry. Mathematical Foundations and Applications, John Wiley and Sons, Chichester 1990. 
[3] J. E. Hutchinson, Fractals and self-similarity, Indiana Univ. Math. J. 30 (1981), 713-747.

[4] S. Kakutani, On equivalence of infinite product measures, Ann. of Math. 49 (1948), 214224.

[5] J. Kigami, Analysis on fractals (Cambridge Tracts in Mathematics 143), Cambridge Univ. Press, Cambridge 2001.

[6] J. Ławrynowicz and L. Wojtczak in cooperation with S. Koshi and O. Suzuki, Stochastical mechanics of particle systems in Clifford-analytical formulation related to Hurwitz pairs of bidimension $(8,5)$, in: Deformations of mathematical structures II. Hurwitz-Type Structures and Applications to Surface Physics, J. Ławrynowicz (ed.), Kluwer Academic, Dordrecht 1994, 213-262.

[7] M. Mori, O.Suzuki, and Y. Watatani, A noncommutative differential geometric method to fractal geometry (I). (Representations of Cuntz algebras of Hausdorff type on fractal sets), Proc. of Int. ISSAC Conf. 1 (2003), 509-518.

[8] L. Nottale, Fractal space-time and microphysics, in: Towards a Theory of Scale Relativity, World Scientific, Singapore 1993.

[9] T. Ogata and O.Suzuki, Mathematical realization of fluctuations, Acta Physicae Superficierum 6 (2004), 81-90.

[10] J.P.Schauder, Zur Theorie stetiger Abbildungen in Funktionalräumen, Math. Zeitschr. 26 (1927), 47-65; reproduced in: J. P. Schauder, Oeuvres, PWN - Éd. Sci. de Pologne, Warszawa 1978, 63-82.

[11] J. P. Schauder, Bemerkungen zu meiner Arbeit "Zur Theorie stetiger Abbildungen in Funktionalräumen”, Math. Zeitschr. 26 (1927), 417-431; reproduced in: J. P. Schauder, Oeuvres, PWN - Éd. Sci. de Pologne, Warszawa 1978, 83-98.

[12] M. Yamaguchi, M. Hata, and J. Kigami, Mathematics on Fractals (in Japanese), Iwanami Publisher, Tokyo 1993. 


\section{TEORYA}

\section{FUNKEYJ ANALITYGZNYCH}

NAPISAE

Dr. JÓZEF KNIAŹ PUZYNA,

PROFESOR UNIWERSYTETU LWOWSKIEGO.

$$
\text { TOM II. }
$$

[Z 121 figurami w tekscie.]

$$
\text { L W Ó W. }
$$

Nakładem autora $z$ zasiłkiem Akademil Umiejętnosei w Krakowie.

Główny skład w księgarni H. Altenberga we Lwowie.

1900.
BXTRAIT DU BULLETIN DE L'ACADEMIE DES SCIENCES DE CRACOYIR

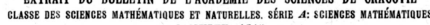

Anwendung der Integralgleichungen zur Bildung der ordinären Differentialgleichungen erster und zweiter Ordnung und der partiellen Differentialgleichungen erster Ordnung

von

J. Puzyna

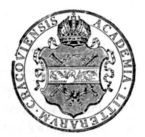

CRACOVIE

IMPRIMERIE DE LUNIVERSITÉ

Geometrisches

in der

\section{Weierstraßschen Theorie}

der algebraischen Funktionen.

Von

Josef v. Puzyna.

Teschen.

K. o. K. Hofbuchdruckerei Karl Prochaska.
Three important publications of Józef kniaź Puzyna 
MONOGRAFJE MATEMATYCZNE KOMITET REDAKCYJNY:

S. BANACH, B. KNASTER, K. K URATOW $S K I$,

S. MAZURKIEWICZ, W. SIERPIŃSKI i H. STEINHAUS TOM I

\section{T H É O R I E}

D E $S$

\section{OPÉRATIONS LINÉAIRES}

$\begin{array}{lll}\mathbf{P} & \mathbf{A} & \mathbf{R}\end{array}$

STEFAN BANACH

PROFESSEUR Aं LUNIVERSITE DE LWOW

$Z$ SUBWENCJI FUNDUSZU KULTURY NARODOWEJ

W A R $S$ Z A W A $19_{9} 3_{2}$
Les „Monographies Mathématiques“ seront publićes en francais, allemand, anglais ou italien en volumes brochés de 150

$$
\text { à } 300 \text { pages in } 8^{\circ} \text {. }
$$

En préparation se trouvent: de M. $S$. $S$ aks, Théorie de

l'Intégrale, de M. K. Kuratowski, Topologie I, et plusieurs autres.

Chaque volume sera livré par la poste franco contre demande adressće directement à

"MONOGRAF JE MATEMATYCZNE" SEMINAR. MATEM. UNIW WARSZ. OCZKI $\mathrm{N}_{\mathrm{r} .} 3$

WARSZAWA (Varsovie), Pologne

et accompagnée de l'envoi du montant par mandat international ou par chèque (ou bien du versement à P. K. O. Nr. 45.177 Prof. Dr. K. Kuratowski „Monografje Matematyczne“, Lwów).

Les volumes seront aussi en vente dans les librairies.

Le prix de ce volume est 3 dollars U. S. A.

Pour les Membres de la Société Polonaise de Mathématique 2 dollars ou $21.17,50$.

\section{MONOGRAFJE MATEMATYCZNE \\ KOMITET REDAKCYJNY:}

S. B A NA CH, B. KNASTER, K. KURATOW $S K I$,

S. MAZURKIEWICZ, W. SIERPIŃSKI i H. STEINHAUS TOM VI
THEORIE

DER

\section{ORTHOGONALREIHEN}

vON

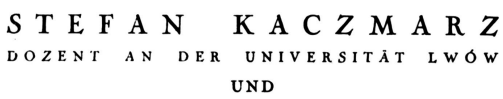

H U G O $S$ T E I N H A U $S$

PROFESSOR AN DER UNIVERSITÁT LWOW
CHELSEA PUBLISHING COMPANY NEW YORK, N.Y.

1951
Vol. I of Monografje Matematyczne - Monographies Mathématiques - title page and announcement of next volumes; and vol. VI - title page of U.S.-reedition 

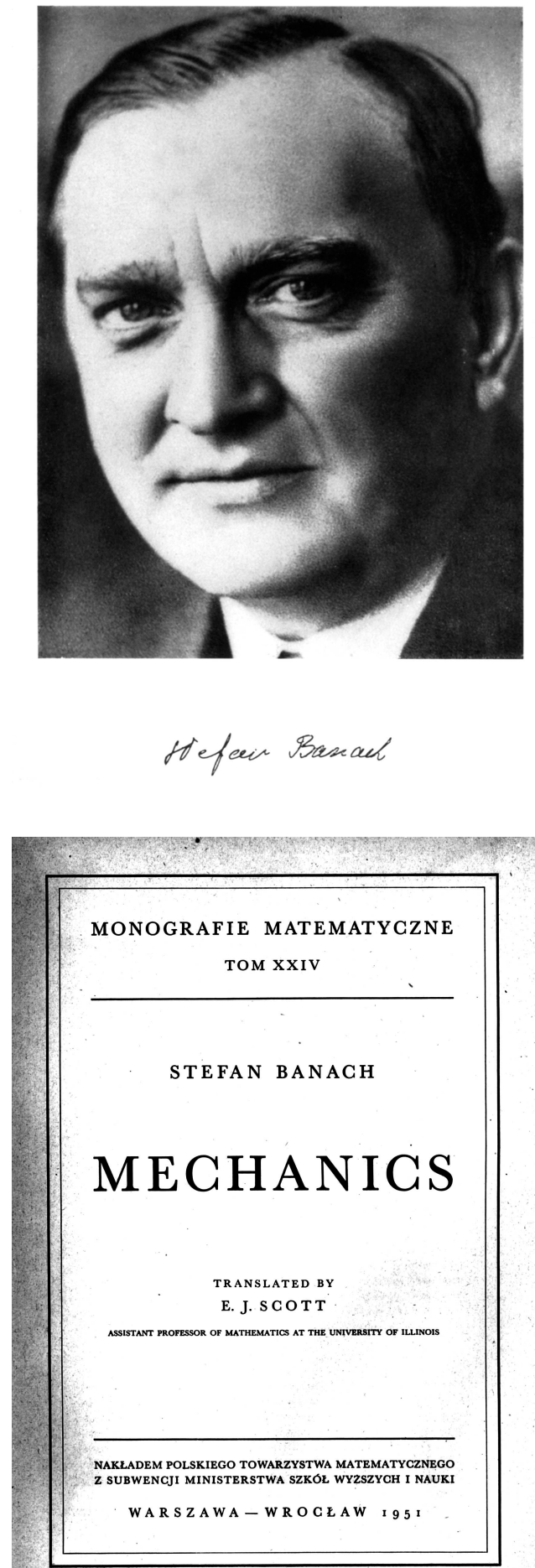
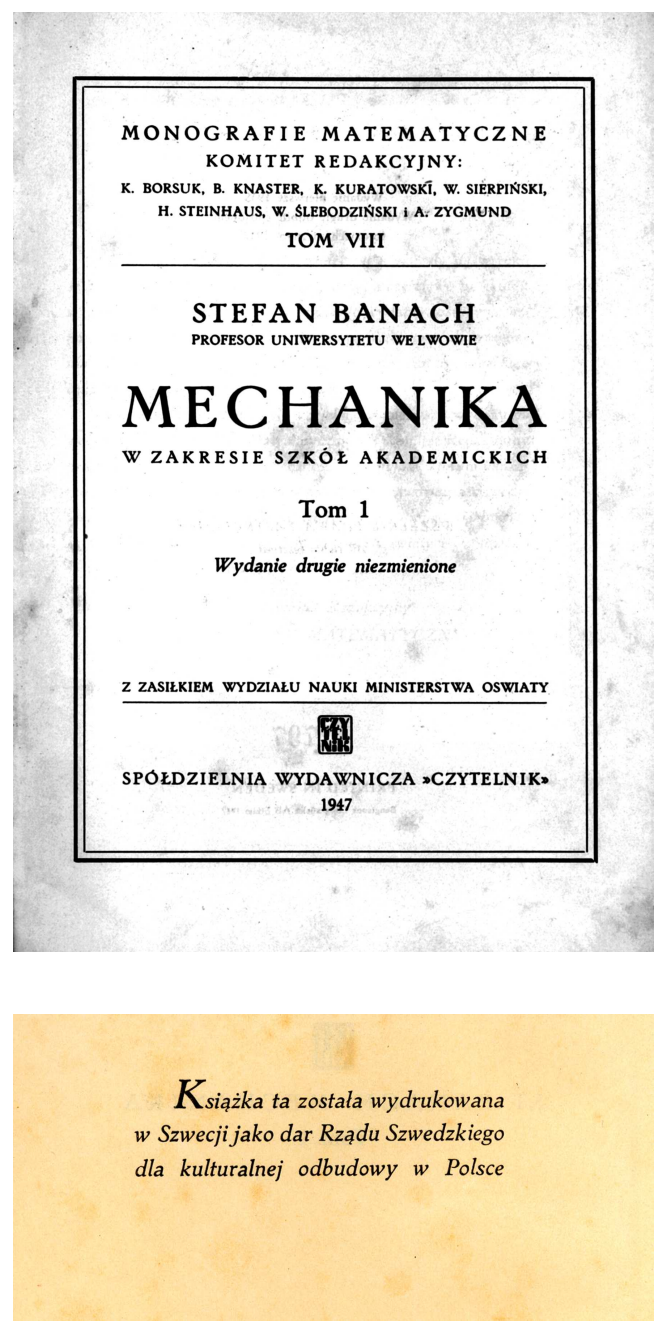

Vol. III of Monografie Matematyczne printed in Sweden as a gift of the Swedish Government, and the English translation of vols. VIII and IX, printed as vol. XXIV of the Monografie 
MONOGRAFIE MATEMATYCZNE

TOM XXV

WŁADYSŁAW NIKLIBORC

RÓWNANIA RÓŻNICZKOWE

CZFŚŚ I

DO DRUKU OPRACOWAE. ZYGMUNT CHARZYŃSKI

NAK $A D E M$ POLSKIEGO TOWARZYSTWA MATEMATYCZNEGO Z SUBWENCJI MINISTERSTWA SZKÓも WYŻSZYCH I NAUKI WA R S A W A - W ROC LAW 195
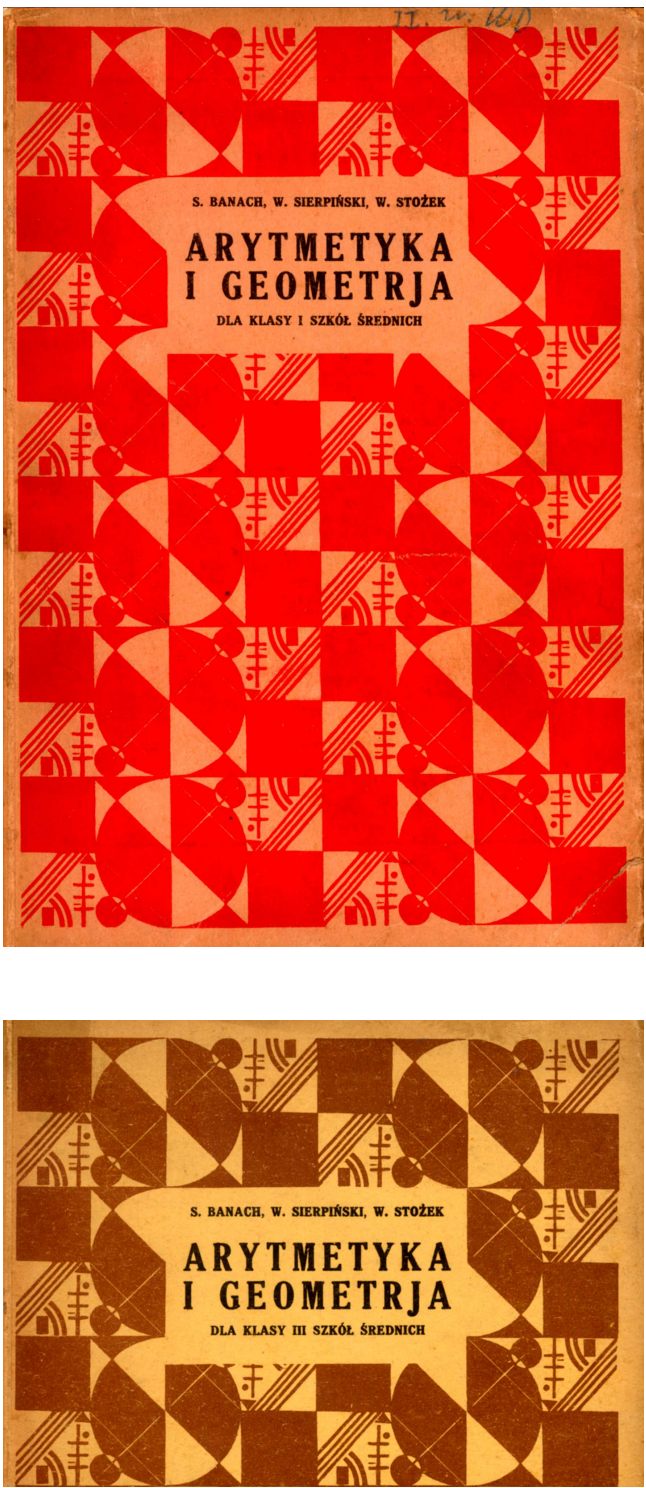

Title page of vol. XXV of Monografie Matematyczne - edition of an unfinished manuscript by Władysław Nikliborc, a victim of the postwar Polish Security Police, and the title pages of textbooks by Stefan Banach, Wacław Sierpiński, and Włodzimierz Stożek 

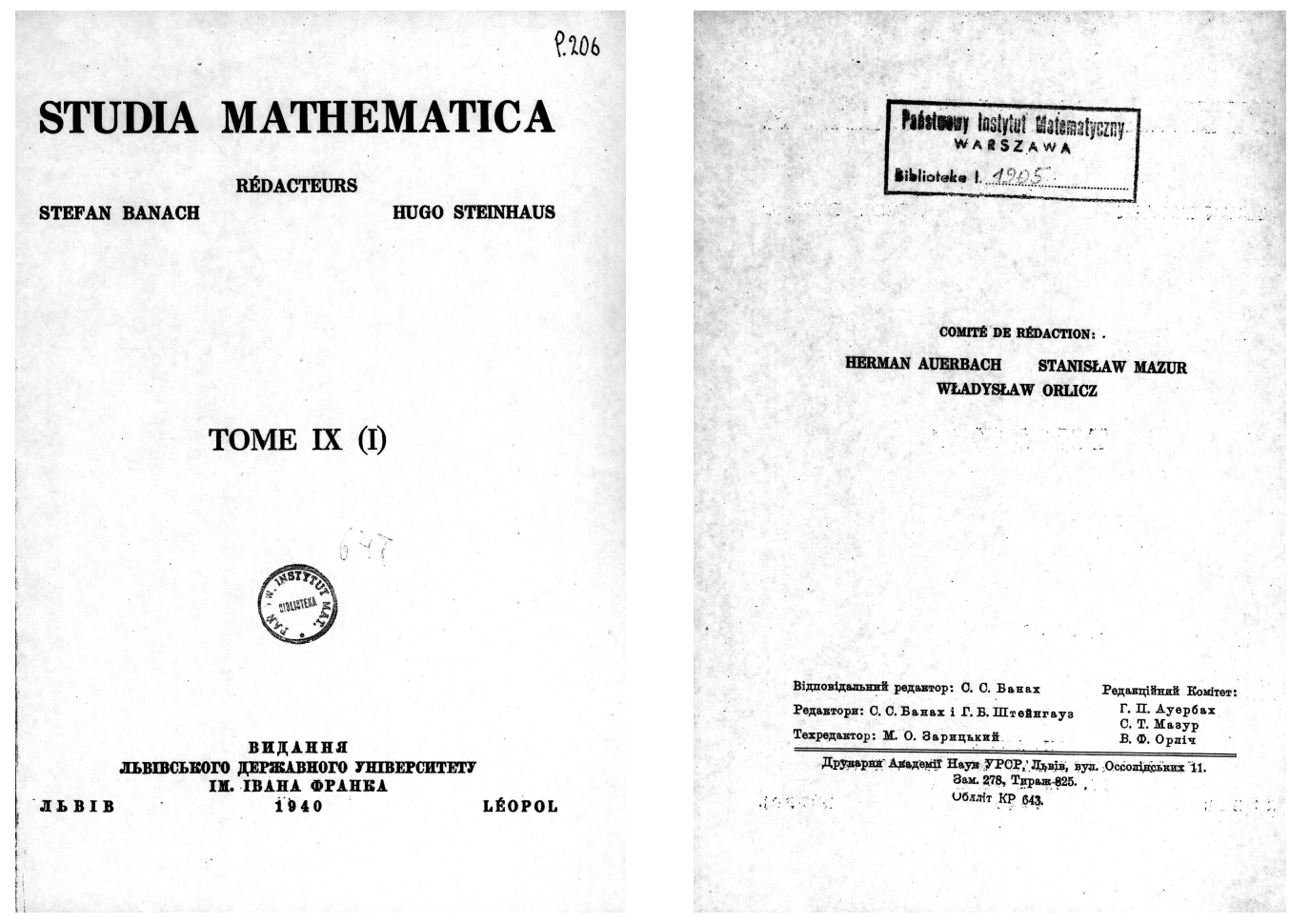

\section{MATEMATUKA}

\section{ДЛЯ І КЛЯСИ СЕРЕДНІХ ЗАГА.ЛЬНО ОСВITHIX IIIKIЛ}

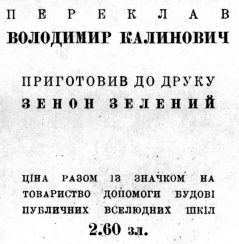

Druk wykonala drukarnio

Towarzystwa Naukowego im. Szewczenki we Lwowie ul. Czarniecklego I. 26 . pod zarzqdem St. Mykitkı. Prace introligatorskie wykonal Józef Legeżynskb we Lwowie.

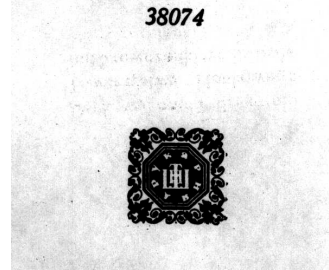

Vol. XII of Studia Mathematica published by the Ivan Franko University in 1940 and the Ukrainian edition of a secondary school textbook by Arnold Frejlich and Władysław Orlicz, published by Polish School Publishers and printed by the Ukrainian Taras Shevchenko Learned Society in 1938 
146

DOCUMENTS

HCurranc.

Tranax Crenan brenakobur, yp. 30. IT. 1992 blhpabobi, nolakh.

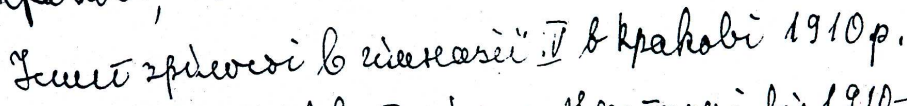

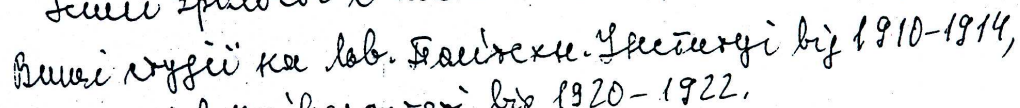
itha lob. Gaibepareri big 1920-1922.

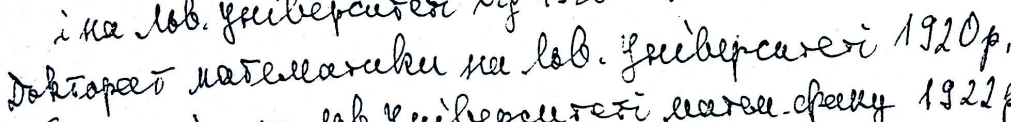

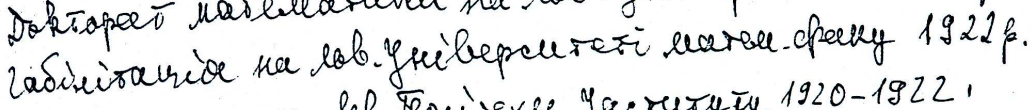

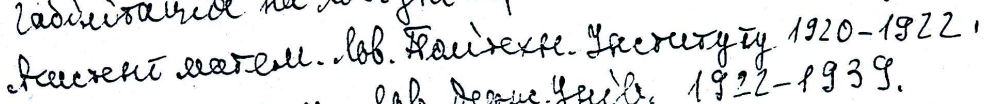

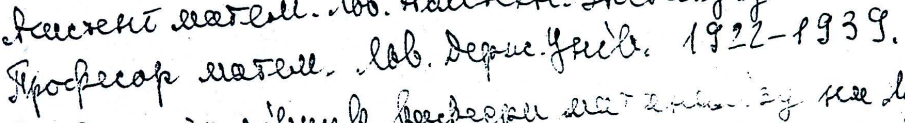

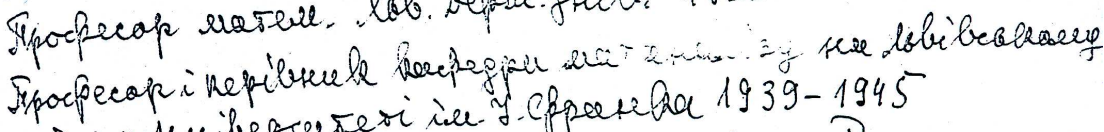

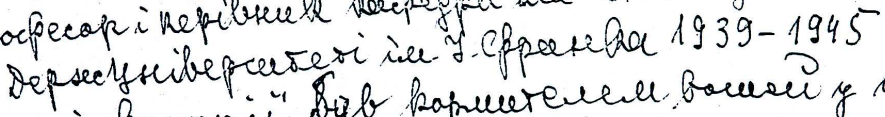

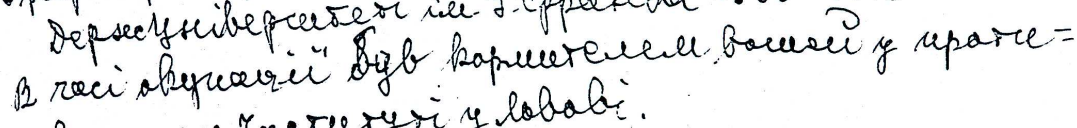
reckossubery Tec tury ri y lobabi.

Trea thagarii hayk b kpanabi bi $1924 \%$.

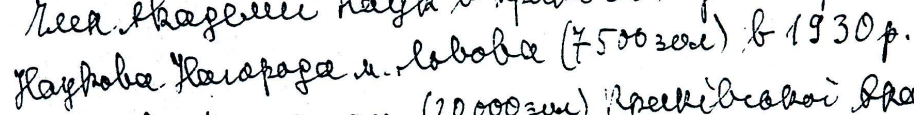

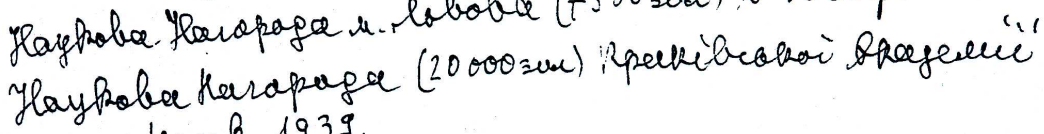
teayk 1939.

Denyrar bieakpagu y lababi by $1940 \mathrm{p}$.

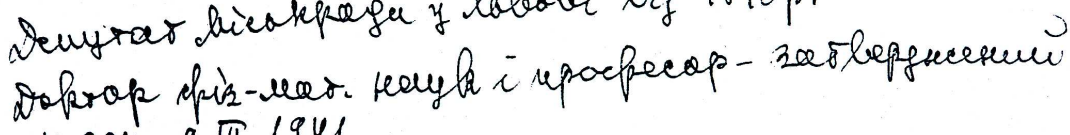

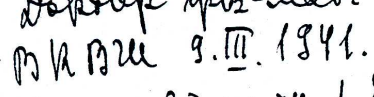

Depare cpizuary $1.2 . y$. by $1939-1944$ p.

lobib, 16. I. 1945

bege. Plepetimaboro 12, be. 4 .

C.C. Travax 